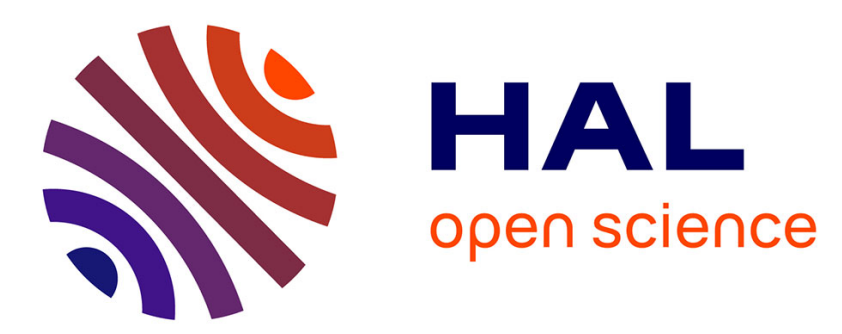

\title{
Importance of metabolic coupling for the dynamics of gene expression following a diauxic shift in $\mathrm{E}$. coli
}

Valentina Baldazzi, Delphine Ropers, Johannes Geiselmann, Daniel Kahn, Hidde de Jong

\section{- To cite this version:}

Valentina Baldazzi, Delphine Ropers, Johannes Geiselmann, Daniel Kahn, Hidde de Jong. Importance of metabolic coupling for the dynamics of gene expression following a diauxic shift in E. coli. [Research Report] RR-7617, INRIA. 2011. inria-00593109

\section{HAL Id: inria-00593109 \\ https://hal.inria.fr/inria-00593109}

Submitted on 13 May 2011

HAL is a multi-disciplinary open access archive for the deposit and dissemination of scientific research documents, whether they are published or not. The documents may come from teaching and research institutions in France or abroad, or from public or private research centers.
L'archive ouverte pluridisciplinaire HAL, est destinée au dépôt et à la diffusion de documents scientifiques de niveau recherche, publiés ou non, émanant des établissements d'enseignement et de recherche français ou étrangers, des laboratoires publics ou privés. 


\title{
Importance of Metabolic Coupling for the Dynamics of Gene Expression Following a Diauxic Shift in E. coli
}

\author{
Valentina Baldazzi — Delphine Ropers — Johannes Geiselmann — Daniel Kahn — \\ Hidde de Jong
}

$\mathbf{N}^{\circ} 7617$

Mai 2011

\section{apport}

de recherche 



\title{
Importance of Metabolic Coupling for the Dynamics of Gene Expression Following a Diauxic Shift in E. coli
}

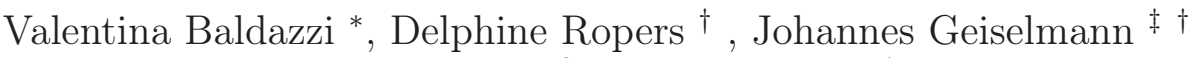 \\ , Daniel Kahn 8 , Hidde de Jong ${ }^{\dagger}$ \\ Theme : \\ Équipe-Projet Ibis \\ Rapport de recherche $\mathrm{n}^{\circ} 7617$ - Mai 2011 - 43 pages
}

\begin{abstract}
Gene regulatory networks consist of direct interactions, but also include indirect interactions mediated by metabolism. We investigate to which extent these indirect interactions arising from metabolic coupling influence the dynamics of the system. To this end, we build a qualitative model of the gene regulatory network controlling carbon assimilation in E. coli, and use this model to study the changes in gene expression following a diauxic shift from glucose to acetate. In particular, we compare the steady-state concentrations of enzymes and transcription regulators during growth on glucose and acetate, as well as the dynamic response of gene expression to the exhaustion of glucose and the subsequent assimilation of acetate. We find significant differences between the dynamics of the system in the absence and presence of metabolic coupling. This shows that interactions arising from metabolic coupling cannot be ignored when studying the dynamics of gene regulatory networks.
\end{abstract}

Key-words: Systems biology, microbiology, qualitative models, gene regulatory networks, carbon metabolism

* INRA, Plantes et Systèmes de Culture Horticoles, Domaine St Paul, Site Agroparc, 84941 Avignon Cedex 9, France.

${ }^{\dagger}$ INRIA Grenoble - Rhône-Alpes, 655 avenue de l'Europe, Montbonnot, 38334 Saint Ismier Cedex, France.

¥ Laboratoire Adaptation et Pathogénie des Microorganismes (CNRS UMR 5163),Université Joseph Fourier, Bâtiment Jean Roget, Faculté de Médecine-Pharmacie, La Tronche, France.

$\S$ Laboratoire de Biométrie et Biologie Evolutive (CNRS UMR 5558), Université Lyon 1, INRA, Villeurbanne, France.

Centre de recherche INRIA Grenoble - Rhône-Alpes 655, avenue de l'Europe, 38334 Montbonnot Saint Ismier Téléphone : +33476615200 - Télécopie +33476615252 


\section{Importance du couplage métabolique pour la dynamique d'expression de gènes suite à une transition diauxique chez $E$. coli}

Résumé : Les réseaux de régulation géniques sont composés d'interactions directes, mais incluent aussi des interactions indirectes dues au couplage avec le métabolisme. Nous étudions dans quelle mesure ces interactions indirectes influencent la dynamique du système. Dans ce but, nous avons construit un modèle qualitatif du réseau de régulation génique contrôlant l'assimilation du carbone chez $E$. coli et nous utilisons ce modèle pour étudier la réponse génique lors d'une diauxie glucose-acetate. Plus précisément, nous comparons les concentrations à l'état stationnaire des enzymes et des régulateurs globaux lors d'une croissance sur glucose et sur acetate, ainsi que la dynamique de l'expression de gènes suite à l'épuisement du glucose. Nous trouvons des différences significatives entre la dynamique prédite en absence et en présence des interactions indirectes. Nos résultats montrent que les interactions dues au couplage avec le métabolisme doivent être prises en compte quand on s'intéresse à la dynamique de réseaux de régulation géniques.

Mots-clés : Biologie de systèmes, microbiologie, modèles qualitatifs, réseaux de régulation géniques, métabolisme carboné 


\section{Introduction}

The reorganization of gene expression patterns in response to environmental changes is controlled by so-called gene regulatory networks, which ensure the coordinated expression of clusters of functionally related genes. The interactions in the network may be direct, as in the case of a gene coding for a transcription factor regulating the expression of another gene. Most of the time, however, regulatory interactions are indirect, e.g. when a gene encodes an enzyme producing a transcriptional effector (Brazhnik et al., 2002). The latter interactions arise from the fact that gene regulation is embedded in a complex, multi-level system that tightly integrates gene expression with metabolism. We call the occurrence of indirect interactions between enzymes and genes, mediated by metabolism, metabolic coupling.

In previous work (Baldazzi et al.,2010), we showed how indirect interactions arising from metabolic coupling can be derived from a model of the underlying biochemical reaction network. We applied this approach to the carbon assimilation network in Escherichia coli. Our results showed that the derived gene regulatory network is densely connected, contrary to what is usually assumed. Moreover, we found that the signs of the indirect interactions are largely fixed by the direction of metabolic fluxes, independently of specific parameter values and rate laws, and that a change in flux direction may invert the sign of indirect interactions. This leads to a feedback structure that is at the same time robust to changes in the kinetic properties of enzymes and that has the flexibility to accommodate radical changes in the environment.

It remains an open question, however, to which extent the indirect interactions induced by metabolic coupling influence the dynamics of the system. This is a key issue for understanding the relative contributions of the regulation of gene expression and metabolism during the adaptation of the cell to changes in its environment. Indirect interactions could be essential in shaping the response of the cell, giving it the required flexibility to adapt to external perturbations. However, one could also argue that indirect interactions only have a fine-tuning effect, simply refining the gene expression levels at which the system would stabilize without metabolic coupling.

In order to decide between these two hypotheses, and obtain a clearer view of the role of metabolic coupling in the adaptation of gene expression, we build a dynamic model of the gene regulatory network controlling carbon assimilation in E. coli, and use this model to study the changes in gene expression following a diauxic shift from glucose to acetate. More specifically, we develop a qualitative model using piecewise-linear (PL) differential equations that allows us to encode the regulatory logic of the system in a simple way (Batt et al., 2008; Glass and Kauffman, 1973). Even though good quantitative models of carbon metabolism in E. coli exist in the literature (Bettenbrock et al., 2005; Chassagnole et al., 2002; Kotte et al., 2010), not much is known about precise mechanisms and parameter values for gene regulation. Moreover, qualitative models are an appropriate tool for analyzing if metabolic coupling can induce major changes in the gene expression dynamics, i.e., will not only have an effect on quantitative but also qualitative properties of the system dynamics.

We build several qualitative models, corresponding to a network topology including all, some, or none of the indirect interactions, respectively. The dynamical properties of the models are analyzed and compared with available 
experimental data. In particular, we compare the steady-state concentration of enzymes and transcription regulators during growth on glucose and acetate, as well as the dynamic response of gene expression to the exhaustion of glucose and the subsequent assimilation of acetate.

We find significant differences between the dynamics of the system in the absence and presence of metabolic coupling. In particular, the indirect interactions are essential for correctly reproducing the observed adaptation of gene expression to a change in carbon source. In particular, indirect intercations mediated by the activation of the regulators Crp and FruR is seen to be key for the adjustment of gene expression levels when changing the carbon source from glucose to acetate.

Our work thus underlines the importance of metabolic coupling in gene regulatory networks, and shows that such indirect interactions cannot be neglected when studying the adaptation of an organism to changes in its environment. 


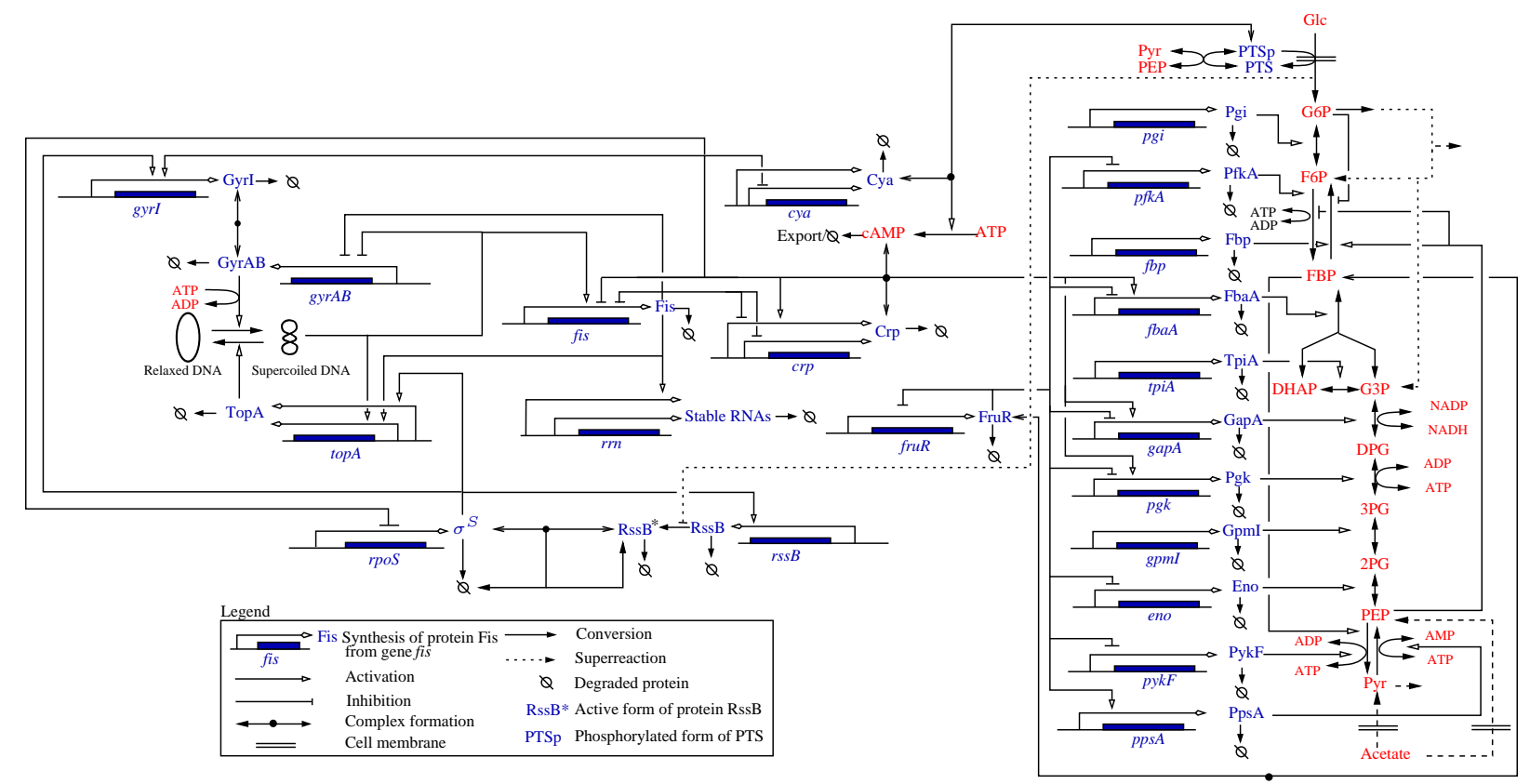

Figure 1: Carbon assimilation network in E. coli, consisting of the glycolysis and gluconeogenesis pathways and their genetic and metabolic regulation (Baldazzi et al., 2010). 


\section{Methods}

\subsection{Metabolic coupling in gene regulatory networks}

In Baldazzi et al. (2010) a mathematical method is proposed, based on a combination of time-scale approximations and sensitivity criteria from metabolic control analysis, to reconstruct the indirect interactions between genes from a model of the underlying network of biochemical reactions. In the following, we briefly summarize the basic principles of this approach.

We start by building a stoichiometry model of the network. The model takes the form of a system of ordinary differential equations (ODEs) and describes the rate of change of the concentrations of the different molecular species in the network:

$$
\dot{x}=N v(x), \quad x(0)=x_{0}
$$

where $x \in \mathbb{R}_{+}^{n}$ denotes the vector of concentrations and $v: \mathbb{R}_{+}^{n} \rightarrow \mathbb{R}^{q}$ the vector of reaction rates. $N \in \mathbb{Z}^{n \times q}$ is a stoichiometry matrix. In the presence of conserved quantities, $N$ is the reduced stoichiometry matrix (Reder, 1988). Contrary to kinetic models, the rate laws are not explicitly specified, but only the functional dependency of the reaction rates on specific molecular species are given. We do not develop the rate laws, because only the signs of the partial derivatives are used for reconstructing the indirect interactions (see below).

As a first step, the model can be simplified by making the quasi-steady-state (QSS) approximation (Heinrich and Schuster, 1996). Two different time-scales are distinguished, one corresponding to the slow processes (protein synthesis and degradation) and one to the fast processes (complex formation and enzymatic reactions). Based on time-scale separation, the original model can be rewritten as two distinct subsystems

$$
\begin{array}{ll}
\dot{x}^{s}=N^{s} v^{s}\left(x^{s}, x^{f}\right), & x^{s}(0)=x_{0}^{s} \\
\dot{x}^{f}=N^{f} v^{f}\left(x^{s}, x^{f}\right), & x^{f}(0)=x_{0}^{f}
\end{array}
$$

where $x^{s} \in \mathbb{R}_{+}^{m}$ and $x^{f} \in \mathbb{R}_{+}^{n-m}$ are vectors of slow and fast variables, respectively, and $N^{s}, N^{f}$ and $v^{s}, v^{f}$ the corresponding stoichiometry matrices and rate vectors. The slow variables describe total concentrations of the proteins, and the fast variables concentrations of metabolites and complexes of proteins and signalling molecules.

The QSS approximation makes the assumption that at the time-scale of the slow processes the fast part of the system is at steady state, instantly adapting to the dynamics of the slow variables, i.e. $N^{f} v^{f}\left(x^{s}, x^{f}\right)=0$ (see Heinrich and Schuster (1996) and Khalil (2001) for the conditions under which the QSS approximation is valid). This means that, after an initial transient, the dynamics of the fast system can be well approximated by an algebraic function of the slow variables, if such a function can be found: $x^{f}=g\left(x^{s}\right)$, $g: \mathbb{R}_{+}^{m} \rightarrow \mathbb{R}_{+}^{n-m}$. The resulting reduced system, at the slow time-scale, takes the following form

$$
\dot{x}^{s}=N^{s} v^{s}\left(x^{s}, g\left(x^{s}\right)\right)
$$

The interest of this model is that it represents the structure of direct and indirect interactions between the slow variables, that is, the structure of the gene 
regulatory network. Since we have not specified the rate laws, and in general these are complex nonlinear functions of the concentration variables, it is not possible to obtain a closed-form expression for the function $g$. We therefore follow another strategy to characterize the indirect interactions between the slow variables, by studying the Jacobian matrix $\mathcal{J} \in \mathbb{R}^{m} \times \mathbb{R}^{m}$ of the system in Eq. 4:

$$
\mathcal{J}=\frac{\partial \dot{x}^{s}}{\partial x^{s}}=N^{s} \frac{\partial v^{s}\left(x^{s}, g\left(x^{s}\right)\right)}{\partial x^{s}}+N^{s} \frac{\partial v^{s}\left(x^{s}, g\left(x^{s}\right)\right)}{\partial x^{f}} \frac{\partial g\left(x^{s}\right)}{\partial x^{s}}
$$

The Jacobian matrix includes the direct effect of each slow variable on the others (first term) and the indirect effect via coupling through the fast system (second term). It accounts for direct regulation of gene expression by transcription factors as well as indirect regulation through metabolic intermediates. Applying the implicit function theorem to the QSS equation, $\partial g\left(x^{s}\right) / \partial x^{s}$ can be further developed as

$$
\frac{\partial g\left(x^{s}\right)}{\partial x^{s}}=-M^{-1} N^{f} \frac{\partial v^{f}\left(x^{s}, x^{f}\right)}{\partial x^{s}}
$$

where $M=N^{f} \partial v^{f}\left(x^{f}, x^{s}\right) / \partial x^{f}$ is the Jacobian matrix of the fast system. Eq. [6 describes the response of the fast system around its steady state to changes in the slow variables. $-M^{-1} N^{f}$ is known as the matrix of (non-normalized) concentration control coefficients in metabolic control analysis (Heinrich and Schuster, 1996).

The symbolic computation of the elements of $\mathcal{J}$ gives rise to complex expressions in terms of elementary partial derivatives of the reaction rates with respect to the concentration variables, called (non-normalized) elasticities (Heinrich and Schuster, 1996). Even in the absence of a precise specification of kinetic rate laws and parameters values, most elasticities have a well-defined sign, given a convention on the positive flux direction. Together with constraints imposed by the stability of the steady state of the fast system, the signs of the elasticities are used to evaluate the signs of the elements of the Jacobian matrix. Notice that the sign of the elasticity of a reversible reaction with respect to its enzyme concentration can be positive or negative, depending on the flux direction. As a consequence, the signs of the elements of $\mathcal{J}$ depend on the growth conditions, as the latter may influence the flux directions, and thus the signs of (some of) the elasticities.

Fig. 2illustrates the application of the method to a small example, describing the synthesis of cyclic AMP (cAMP) and its effect on gene expression in response to glucose depletion. Panels $A$ and $B$ show the reactions playing a role in this subsystem and the stoichiometry model obtained by separating the time-scales for the expression of the genes fis, cya, and crp on one hand, and for cAMP synthesis and the formation of the complex Crp.cAMP on the other. A graphical representation of the reduced network, displaying the regulatory interactions between the slow variables of the system, is shown in panel $C$. Some of the interactions are direct, such as those between Fis and its targets. However, many of the interactions are indirect, in particular the influence of Crp, Cya, and external glucose on the metabolic coupling species Crp.cAMP, which in turn controls the expression of the genes in the network. The results for this example are intuitive and easy to derive, but this is no longer the case when large networks like that shown in Fig. 1 are studied. The expressions for the elements of the Jacobian matrix become complex and computer algebra tools may be necessary to resolve their signs (Baldazzi et al, 2010). 


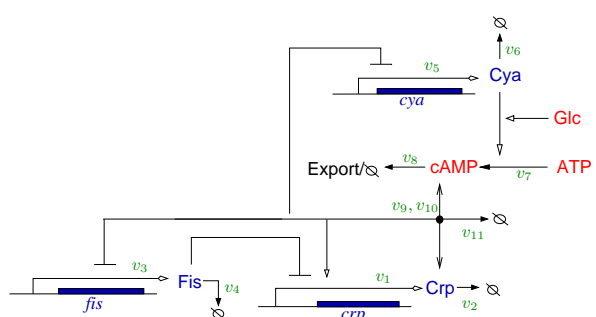

$A$

$$
\begin{gathered}
{\left[\begin{array}{c}
\dot{x}_{C r p} \\
\dot{x}_{F i s} \\
\dot{x}_{C y a}
\end{array}\right]=\left[\begin{array}{cccccc}
1 & -1 & 0 & 0 & 0 & 0 \\
0 & 0 & 1 & -1 & 0 & 0 \\
0 & 0 & 0 & 0 & 1 & -1
\end{array}\right]\left[\begin{array}{c}
v_{1}\left(x_{F i s}, x_{C r p \cdot c A M P}\right) \\
v_{2}\left(x_{C r p}\right) \\
v_{3}\left(x_{C r_{p} \cdot c A M P}, x_{F i s}\right) \\
v_{4}\left(x_{F i s}\right) \\
v_{5}\left(x_{C r p \cdot c A M P}\right) \\
v_{6}\left(x_{C y a}\right)
\end{array}\right]} \\
{\left[\begin{array}{c}
\dot{x}_{C A M P} \\
\dot{x}_{C r p \cdot c A M P}
\end{array}\right]=\left[\begin{array}{ccccc}
1 & -1 & -1 & 1 & 0 \\
0 & 0 & 1 & -1 & -1
\end{array}\right]\left[\begin{array}{c}
v_{7}\left(x_{C y a}, u_{G l c}\right) \\
v_{8}\left(x_{c A M P}\right) \\
v_{9}\left(x_{C A M P}, x_{f r e e C r p}\right) \\
v_{10}\left(x_{C r p \cdot c A M P}\right) \\
v_{11}\left(x_{C r p \cdot c A M P}\right)
\end{array}\right]} \\
B
\end{gathered}
$$

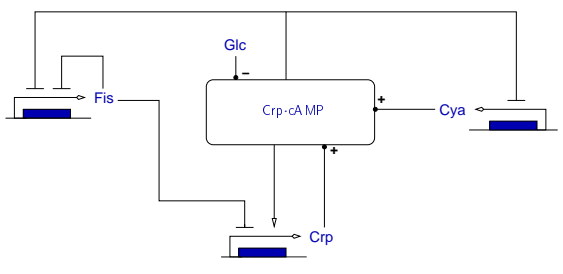

$$
\mathcal{J}=\left[\begin{array}{ccc}
+ & - & - \\
- & - & 0 \\
+ & 0 & -
\end{array}\right]
$$

C

Figure 2: Example of the derivation of a gene regulatory network, for a subnetwork involving the activation of the transcription regulator Crp. A: Network diagram (see Fig. 1 for the legend). The reactions correspond to protein synthesis $\left(v_{1}, v_{3}, v_{5}\right)$, protein degradation $\left(v_{2}, v_{4}, v_{6}, v_{11}\right)$, enzymatic reactions $\left(v_{5}\right)$, complex formation $\left(v_{9}, v_{10}\right)$, and export $\left(v_{11}\right)$. The synthesis of the second messenger cAMP, and its regulation through the activation of adenylate cyclase Cya under conditions of glucose depletion, are summarized in a single reaction $\left(v_{7}\right)$. Proteins are shown in red, metabolites in blue, and reactions in green. $B$ : Stoichiometry model of the network, with an explicit separation of time-scales following the distinction between slow reactions (protein synthesis and degradation) and fast reactions (enzymatic reactions and complex formation). The slow variables are the total concentrations of Crp $\left(x_{C r p}\right)$, Fis $\left(x_{F i s}\right)$, and Cya $\left(x_{C y a}\right)$, while the fast variables are the concentrations of cAMP $\left(x_{C A M P}\right)$ and Crp.cAMP $\left(x_{C r p \cdot c A M P}\right)$. The glucose concentration is an input variable $\left(u_{G l c}\right)$. For simplicity, ATP and external cAMP are not explicitly modeled in reactions $v_{7}$ and $v_{8}$. $C$ : Derived gene regulatory network showing direct and indirect interactions between genes and their products, obtained from the stoichiometry model in $A$. The concentrations of Crp, Cya, and glucose influence the concentration of the fast coupling species Crp·cAMP, which in turn affects the expression of the three genes in the network ( $i s$, cya, and crp). In addition, the diagram shows direct interactions, such as the regulation of the expression of fis and $\operatorname{crp}$ by Fis. D: Jacobian matrix showing the sign of the derived regulatory interactions between the slow variables $\left[x_{C r p}, x_{F i s}, x_{C y a}\right]^{\prime}$ of the system. 


\subsection{Including metabolic coupling in dynamic models of gene regulatory networks}

Modeling the dynamics of a gene regulatory network amounts to specifying the right-hand side of Eq. 4. However, recall from Sec. 2.1 that we have not fully specified the rate laws. Analysis of Eq. 5, defining the Jacobian matrix $\mathcal{J}$ and thus the structure of the gene regulatory network, provides information that can be exploited for qualitatively modeling the network dynamics. By making the common assumption that gene regulatory interactions are monotone, at least over large concentration range, and sigmoidal (de Jong, 2002; Polynikis et al., 2009), we show how approximate functions for $v^{s}$ can be defined that are suitable for the qualitative analysis of the network dynamics. Numerical simulation studies have shown that, in comparison with conventional kinetic models with fully specified rate laws, the approximations are generally able to preserve the (qualitative) dynamics of the network (Davidich and Bornholdt, 2008; Ropers et al., 2011).

Consider first the case of direct interactions, and more specifically the case $v_{i}^{s}\left(x^{s}, x^{f}\right)=v_{i}^{s}\left(x_{j}^{s}\right)$, for some reaction $i$ and slow variable $x_{j}^{s}$. Typically, $v_{i}^{s}$ represents a protein synthesis rate and $x_{j}^{s}$ the (total) concentration of a transcription factor. Monotonicity of $v_{i}^{s}$ means that $\partial v_{i}^{s} / \partial x_{j}^{s}$ is either positive or negative, depending on the sign in $\mathcal{J}$ (if the sign is positive, then the transcription factor is an activator; if negative, an inhibitor). Sigmoidality of $v_{i}^{s}$ means that $\partial^{2} v_{i}^{s} /\left(\partial x_{j}^{s}\right)^{2}$ has a (single) zero. A simple function that is both monotone and sigmoidal is the Hill function (Plahte et al., 1998; Wittmann et al., 2009):

$$
v_{i}^{s}\left(x_{j}^{s}\right)=\kappa_{i} h^{+}\left(x_{j}^{s}\right)=\kappa_{i} \frac{\left(x_{j}^{s}\right)^{n}}{\left(x_{j}^{s}\right)^{n}+\theta_{j}^{n}} \text { or } v_{i}^{s}\left(x_{j}^{s}\right)=\kappa_{i} h^{-}\left(x_{j}^{s}\right)=\kappa_{i}\left(1-h^{+}\left(x_{j}^{s}\right)\right)
$$

where $\theta_{j}$ is a so-called threshold concentration for $x_{j}^{s}$, and $n$ a cooperativity constant that determines the steepness of the sigmoidal functions. When several transcription regulators directly regulate the expression of a gene, that is, $v_{i}^{s}\left(x^{s}, x^{f}\right)=v_{i}^{s}\left(\ldots, x_{j-1}^{s}, x_{j}^{s}, x_{j+1}^{s}, \ldots\right)$, the possibly combinatorial effect of these regulators can be defined by means of sums, subtractions, and products of Hill functions (Plahte et al., 1998; Wittmann et al., 2009).

Can metabolic coupling also be represented by expressions of Hill functions? Consider the case of an indirect interaction $v_{i}^{s}\left(x^{s}, x^{f}\right)=v_{i}^{s}\left(x_{k}^{f}\right)$ with $x_{k}^{f}=$ $g_{k}\left(x_{j}^{s}\right)$, for some fast variable $x_{k}^{f}$. For example, $x_{j}^{s}$ might represent an enzyme concentration and $x_{k}^{f}$ the concentration of a transcription factor activated by a metabolite whose concentration is under the control of $x_{j}^{s}$. The above-mentioned assumptions of monotonicity and sigmoidality imply that $\partial v_{i}^{s} / \partial x_{k}^{f}$ has a definite sign and that $\partial^{2} v_{i}^{s} /\left(\partial x_{k}^{f}\right)^{2}$ crosses zero (once). By Eq. 5 and the chain rule, the partial derivatives characterizing the indirect effect of the slow variables can be expressed as follows: 


$$
\begin{aligned}
\frac{\partial v_{i}^{s}}{\partial x_{j}^{s}} & =\frac{\partial v_{i}^{s}}{\partial x_{k}^{f}} \frac{\partial g_{k}\left(x_{j}^{s}\right)}{\partial x_{j}^{s}} \\
\frac{\partial^{2} v_{i}^{s}}{\left(\partial x_{j}^{s}\right)^{2}} & =\frac{\partial^{2} v_{i}^{s}}{\left(\partial x_{k}^{f}\right)^{2}}\left(\frac{\partial g_{k}\left(x_{j}^{s}\right)}{\partial x_{j}^{s}}\right)^{2}+\frac{\partial v_{i}^{s}}{\partial x_{k}^{f}} \frac{\partial^{2} g_{k}\left(x_{j}^{s}\right)}{\left(\partial x_{j}^{s}\right)^{2}}
\end{aligned}
$$

Interestingly, from these expressions conditions for the monotonicity and sigmoidality of indirect interactions can be derived. For $v_{i}^{s}$ to be monotone, $\partial g_{k}\left(x_{j}^{s}\right) / \partial x_{j}^{s}$ needs to have a definite sign, at least over a concentration range of interest. The computation of the control coefficients for the E. coli carbon assimilation network, which were shown to generally have a definite sign (Baldazzi et al., 2010), suggests that this condition is often satisfied. As to the sigmoidal dependence of $v_{i}^{s}$ on $x_{j}^{s}$, the first term in Eq. 9 is guaranteed to cross zero, since $\partial^{2} v_{i}^{s} /\left(\partial x_{k}^{f}\right)^{2}$ does and $\left(\partial g_{k}\left(x_{j}^{s}\right) / \partial x_{j}^{s}\right)^{2}$ is a positive multiplicative factor. The sum of the two terms does not necessarily have a sigmoidal shape though, for instance when the second term has very large positive or negative values and dominates the first term. By turning the argument around, however, a sufficient condition can be formulated: the indirect interaction is sigmoidal when the second term is negligible with respect to the first term. This condition is notably satisfied when, over the concentration range of interest, the fast variables are linear or quasi-linear functions of the slow variables, in which case $\partial^{2} g_{k}\left(x_{j}^{s}\right) /\left(\partial x_{j}^{s}\right)^{2}$ equals or approaches 0 .

The above argument, which can be generalized to the case that $g$ is a multivariate function, i.e., $x_{k}^{f}=g_{k}\left(\ldots, x_{j-1}^{s}, x_{j}^{s}, x_{j+1}^{s}, \ldots\right)$, is illustrated in Fig. 3 by means of an indirect interaction in the subnetwork describing the activation of the transcription factor Crp. We consider the control of the crp synthesis rate by Crp and Cya in the absence of glucose, through their effect on the concentration of Crp.cAMP. We have developed a kinetic model with plausible parameter values derived from literature, separated the fast and slow variables as described in Fig. 2, and derived an analytical expression for the function $g$ (see Appendix $\mathrm{A}$ ). As can be seen, the $\operatorname{crp}$ synthesis rate depends on the concentrations of Crp and Cya in a sigmoidal way, and can be modeled by combination of Hill functions.

The above analysis, while feasible for a small subnetwork, can no longer be carried out for metabolic coupling in large networks like the one shown in Fig. 1. due to the difficulty of explicitly solving for $g$. We therefore assume that just like direct interactions, indirect interactions are generally monotone and sigmoidal, and can be modeled by combinations of Hill functions. This has been done before, implicitly and with generally good results, in previous examples of the modeling of gene regulatory networks (Batt et al., 2010; de Jong et al., 2004a; Ropers et al., 2006).

\subsection{Qualitative modeling of gene regulatory networks}

In order to study the role and the relevance of the indirect interactions for the dynamics of the system, in particular its response to environmental changes, we adopt a qualitative modelling approach. More specifically, we use piecewiselinear $(P L)$ differential equations to model regulatory interactions between genes and tools developed for the qualitative analysis of these models. The PL models, 


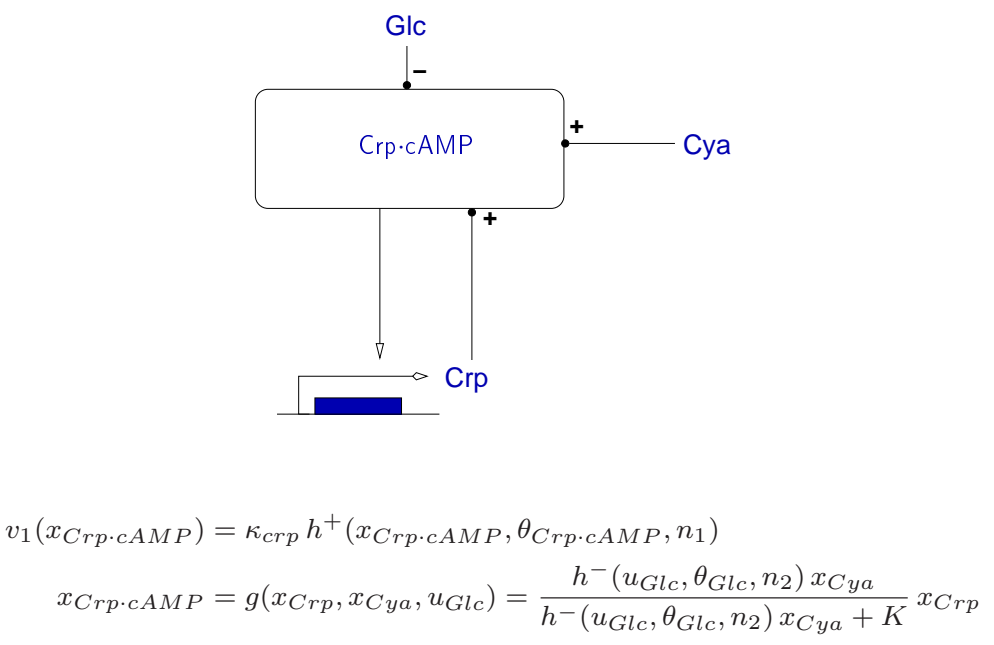

$A$

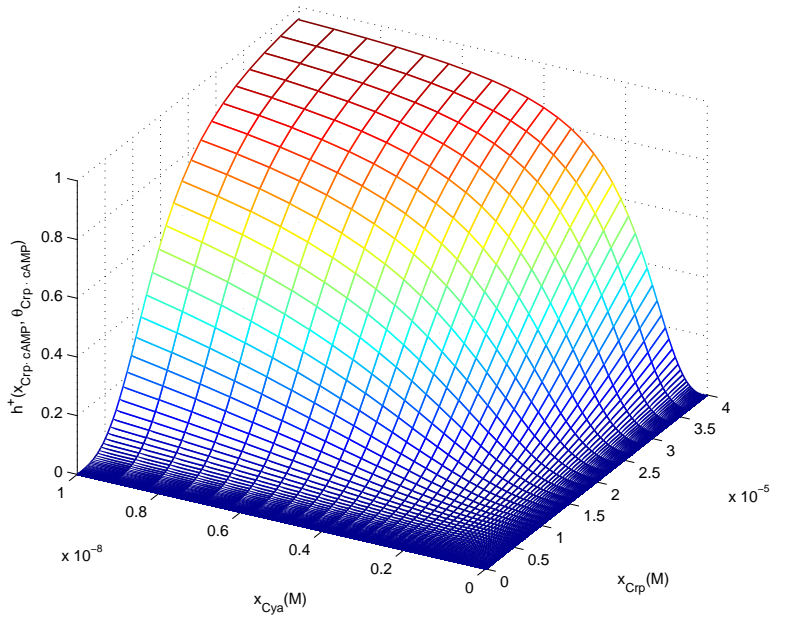

$v_{1}\left(x_{C r p}, x_{C y a}, u_{G l c}\right)=\kappa_{c r p} h^{-}\left(u_{G l c}, \theta_{G l c}, n_{2}\right) h^{+}\left(x_{C r p}, \theta_{C r p}, n_{3}\right) h^{+}\left(x_{C y a}, \theta_{C y a}, n_{4}\right)$

$B$

Figure 3: Example of modeling an indirect interaction in the Crp activation network of Fig. 2. A: Regulation of crp expression by Crp, Cya, and glucose through metabolic coupling, with an explicit model of the indirect interaction. The expression for $v_{1}$ is obtained by solving the QSS equation in a kinetic model of the Crp activation network (Appendix $\mathrm{A}$ ). $\theta_{\text {Crp.cAMP }}$ and $\theta_{\text {Glc }}$ denote threshold concentrations, $\kappa_{c r p}$ a synthesis parameter, $n_{1}$ and $n_{2}$ cooperativity constants, and $K$ a (lumped) kinetic parameter. $B$ : Plot of $h^{+}\left(x_{C r p \cdot c A M P}, \theta_{C r p \cdot c A M P}, n_{1}\right)$ as a function of $x_{C r p}$ and $x_{C y a}$, in case that $h^{-}\left(u_{G l c}, \theta_{G l c}, n_{2}\right) \approx 1$. The resulting sigmoidal surface, representing the synthesis rate of crp, can be approximated by fitting a multiplicative expression of Hill functions, where $\theta_{C r p}$ and $\theta_{C y a}$ are threshold concentrations, and $n_{3}$ and $n_{4}$ cooperativity constants. 
introduced by Glass and Kauffman (1973), are based on the use of step-function approximations of the Hill functions describing direct and indirect regulatory interactions (Sec. 2.2). Here we informally summarize the basic notions underlying the modeling of gene regulatory networks by means of PL models, while mathematical details can be found elsewhere (Batt et al., 2008; de Jong et al., 2004b).

PL models are directly obtained from the dynamics models discussed in the previous section by replacing the Hill functions in the rate laws in the right-hand

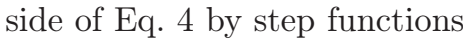

$$
\begin{aligned}
& s^{+}\left(x_{j}^{s}, \theta_{j}\right)= \begin{cases}1, & \text { if } x_{j}^{s}>\theta_{j} \\
0, & \text { if } x_{j}^{s}<\theta_{j}\end{cases} \\
& s^{-}\left(x_{j}^{s}, \theta_{j}\right)=1-s^{+}\left(x_{j}^{s}, \theta_{j}\right)
\end{aligned}
$$

In comparison with Hill functions, step functions discontinuously change their value at the threshold concentration. However, notice that this approximation preserves the switch-like and combinatorial character of gene regulation. On the formal level, the PL models are related to logical models of regulatory networks (see Fauré and Thieffrv (2009); Morris et al. (2010) for reviews on logical models and Snoussi (1989); Wittmann et al. (2009) for a discussion of the relation between logical and PL models).

An advantage of the use of step functions is that they facilitate the analysis of the qualitative dynamics of the PL models, even in higher-dimensional systems (Kappler et al., 2003). The thresholds of the concentration variables define a rectangular partition of the state space, such that in every region not located on a threshold, the PL model reduces to an analytically solvable system of differential equations. Moreover, in every such region the derivatives (trends) of the concentration variables have a determinate sign, which is shown to be invariant for rather weak constraints on the parameter values. The definition of these constraints can generally be inferred from available data in the experimental literature or by intuitive reasoning, even in the absence of quantitative information on parameter values.

The dynamics of PL models is conveniently described by a so-called state transition graph. The states in the graph correspond to the regions in the state space. The transitions between the states arise from solutions of the PL model that enter one region from another. Each transition thus corresponds to a discrete event, namely the crossing of a threshold by one or more concentration variables, possibly entailing a change in the derivative of these variables. The state transition graph can be inferred from the model using the above-mentioned constraints on parameter values rather than exact numerical values. Dynamic properties of the network, such as the stability of steady states, the existence of limit cycles, and the reachability of certain states of interest from a given initial state can be formulated in terms of the graph topology. Moreover, large graphs can be conveniently and efficiently analyzed using model-checking tools.

Genetic Network Analyzer (GNA) is a computer tool specifically developed for the analysis of gene regulatory networks by means of PL models (de Jong et al., 2003; de Jong and Page, 2008; Monteiro et al., 2008). It allows the user to define a regulatory network, build a model of this network, determine the steady states of the system, generate a state transition graph 
starting from an initial state (qualitative simulation of the network dynamics), and analyze the latter graph using model-checking tools. GNA has been used for the analysis of a number of bacterial regulatory networks (de Jong et al., 2004a; Usseglio Viretta and Fussenegger,, 2004; Sepulchre et al., 2007; Ropers et al.,, 2006).

\section{Results and discussion}

\subsection{Metabolic coupling in network controlling carbon as- similation in $E$. coli}

Fig. 1]shows a part of the network involved in the assimilation of carbon sources in E. coli. It consists of the glycolytic and gluconeogenic pathways, the phosphotransferase system, the genes coding for the enzymes, their key transcriptional regulators (Crp, FruR, and Fis), as well as other global regulators of transcription (RpoS, DNA supercoiling, ...). We distinguish two different growth conditions: growth on the carbon-rich substrate glucose (glycolysis) and growth on the carbon-poor substrate acetate (gluconeogenesis). Since glucose and acetate are utilized by distinct metabolic pathways, the flux distributions during glycolysis and gluconeogenesis are significantly different. A diauxic shift from glucose to acetate notably leads to the inversion of glycolytic fluxes (Oh et al., 2002).

The network of biochemical reactions in Fig. 1 1 can be transformed into a network of direct and indirect regulatory interactions following the method outlined in Sec. 2.1. To this end, we use a stoichiometry model of the network describing the dependence of the reaction rates on the substrates, products, enzymes, transcription factors, and other molecular species (Baldazzi et al., 2010). In the case of reversible reactions, the signs of the elasticities, and therefore the signs of the indirect interactions, depend on the directions of the metabolic fluxes. We therefore consider the cases of glycolysis and gluconeogenesis separately.

Fig. $4 A$ shows the resulting network for glycolysis, called $\mathcal{M}_{\text {glyco }}$. The boxes represent so-called coupling species, which channel indirect influences on gene expression through metabolism. For instance, the concentration of Crp.cAMP is modulated by Cya, Crp, and the glycolytic enzymes. Via this route, the glycolytic enzymes indirectly regulate targets of Crp·cAMP. Apart from Crp.cAMP, the network includes three other coupling species: free FruR, RpoS·RssB, and DNA supercoiling. The gene regulatory network for gluconeogenesis is shown in Fig. $4 B$. Some indirect interactions appear in one growth condition and disappear in the other, such as the control of the concentration of free FruR, and its targets, by PykF and PpsA. Moreover, the same interaction may have an opposite sign in the two cases, for instance the effect of GapA on the concentration of free FruR. Metabolic coupling thus allows the structure of regulatory interactions to be dynamically rewired by changes in the environment (Baldazzi et al., 2010).

The networks in Fig. 廿 have been simplified by leaving out some of the glycolytic enzymes. We notably omit enzymes whose expression does not significantly change between growth on glucose and acetate (Oh et al., 2002), enzymes that are constitutively expressed, and enzymes that do not regulate any other gene in the network. Moreover, we keep only a single representative of each group of enzymes with common regulators. This leaves us with GapA, PpsA, 

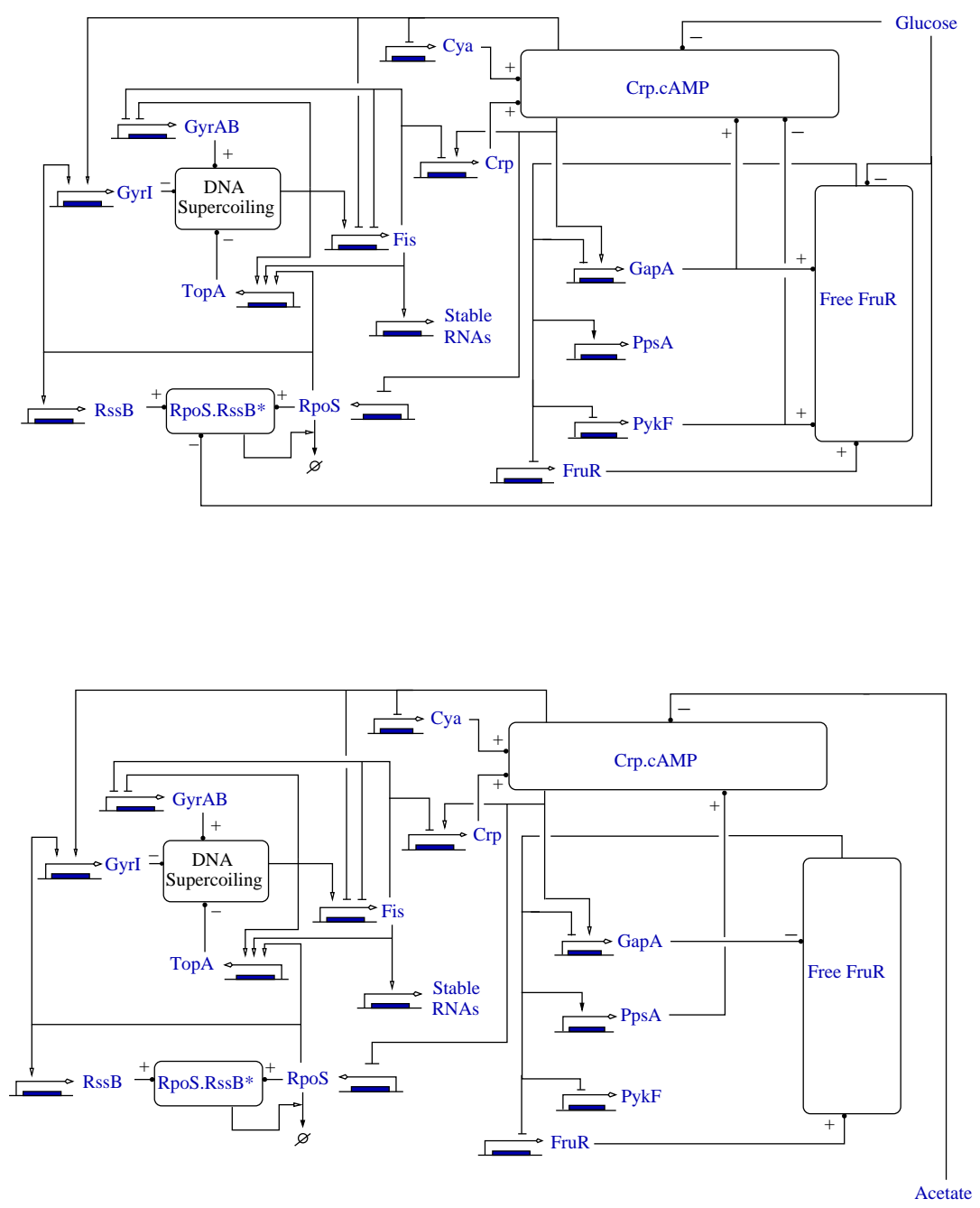

Figure 4: Gene regulatory networks for the glycolytic $(A)$ and gluconeogenic case $(B)$, obtained from the network of biochemical reactions shown in Fig. 1, The networks are denoted by $\mathcal{M}_{\text {glyco }}$ and $\mathcal{M}_{\text {neo }}$, respectively. The boxes indicate the coupling species, here Crp·cAMP, free FruR, RpoS·RssB, and DNA supercoiling. 
and PykF, whose expression patterns are known to characterize the two modes of carbon catabolism considered here (Oh et al., 2002). Notice that these simplifications of the network tend to reduce the number of indirect interactions, and thus their influence on the network dynamics. If an effect of the indirect interactions on the qualitative dynamics is visible in the simplified networks, this will a fortiori be the case in the extended networks as well.

In order to precisely assess the effect of the indirect interactions on the network dynamics, we additionally define a number of reference networks in which all indirect interactions mediated by the coupling species Crp.cAMP and free FruR are systematically removed. The reference networks are called $\mathcal{M}_{\text {glyco/Crp } \cdot c A M P}^{0}, \mathcal{M}_{\text {neo/Crp.cAMP }}^{0}, \mathcal{M}_{\text {glyco/free FruR }}^{0}$, and $\mathcal{M}_{\text {neo/free FruR }}^{0}$ As an extreme case, a network consisting of transcriptional regulatory interactions only is also considered. This last network is simply called $\mathcal{M}^{0}$, and is the same for glycolysis and gluconeogenesis. The topology of $\mathcal{M}^{0}$ networks is shown in Fig 5 (glycolysis) and Appendix C (gluconeogenesis).

We remark that when omitting indirect interactions, we make an exception for transcriptional regulatory interactions, even when the control they exert requires a metabolic effector (e.g., cAMP for Crp). Although not direct in

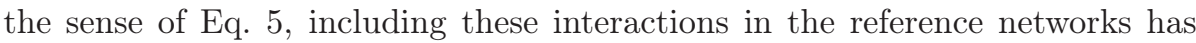
the advantage of complying with the usual notion of transcriptional regulatory networks employed in curated databases (Keseler et al., 2009). As above, the approach is conservative, in the sense that any differences in the qualitative dynamics of the networks with and without metabolic coupling will be exacerbated under a stricter definition of (in)direct interactions.

\subsection{Qualitative modeling of carbon assimilation network}

The networks shown in Fig. 4 and 5 are gene regulatory networks, in the sense that they describe the (direct or indirect) influence of the products of one gene on the expression of another gene (Bolouri, 2008). The interactions can be positive (activation) or negative (inhibition). A variety of methods exist to model and analyze the dynamics of gene regulatory networks (Karlebach and Shamir, 2008). Here we focus on the use of qualitative models, which provide a coarsegrained description of the dynamics of gene expression, in the sense that they do not explicitly specify the biochemical mechanisms. However, they include the logic of gene regulation and allow different expression levels of the genes to be distinguished. They are interesting in their own right, as a way to capture in a simple manner the complex dynamics of a large regulatory network without quantitative data (de Jong and Ropers, 2006; Morris et al., 2010). However, they can also be used as a first step to orient the development of more detailed quantitative ODE models.

More specifically, we use so-called piecewise-linear (PL) differential equations, a formalism originally introduced by Glass and Kauffman (1973) for describing gene regulatory networks (Sec. 2.3). PL models allow the qualitative dynamics of large regulatory networks to be analyzed using inequality constraints on parameters rather than exact numerical values. The network structures in Fig. 4 and 5 are transformed into qualitative PL models by defining the differential equations for each of the gene products (protein concentrations) as well as parameter constraints that characterize the system dynamics. Intuitively, this amounts to specifying the logic of the regulation of the genes and the relative 

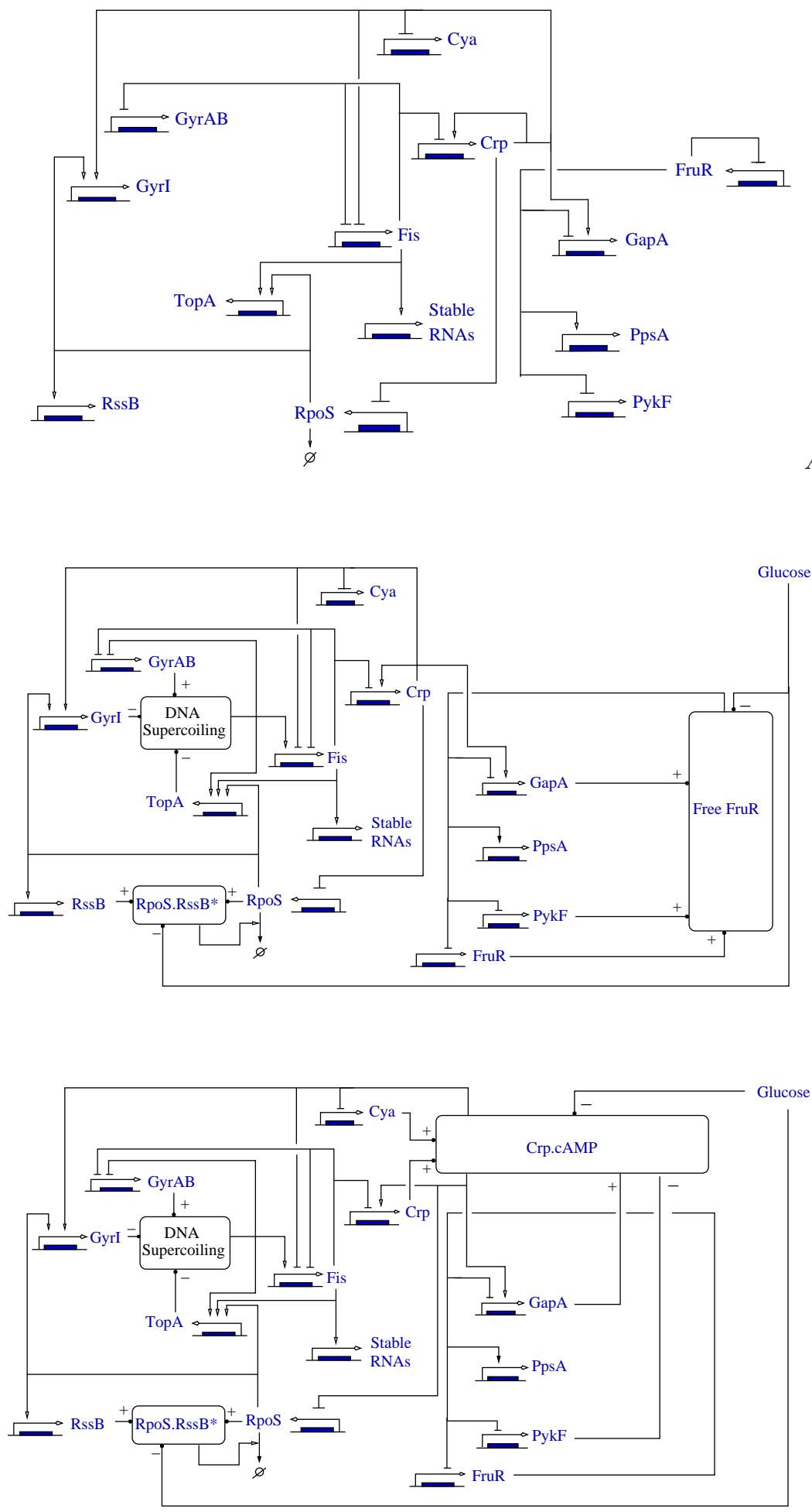

C

Figure 5: Transcriptional regulatory networks $A: \mathcal{M}^{0}, B: \mathcal{M}_{\text {glyco/Crp.cAMP }}^{0}$, and $C: \mathcal{M}_{\text {glyco/free FruR }}^{0}$, obtained by omitting the indirect interactions in Fig. 4 , RR $n^{\circ} 7617$ 
strength of the different interactions regulating the expression of a gene. Recall that the PL models for the $\mathcal{M}_{\text {glyco }}$ and $\mathcal{M}_{\text {neo }}$ networks are slightly different, due to differences in the network topology in glycolytic and gluconeogenic growth conditions (Sec. 3.1). The PL models extend an existing qualitative model of the network of global regulators in E. coli (Ropers et al., 2006), by adding metabolic coupling induced by carbon catabolism. The following principles have guided the modeling of the network:

- The existence of a single stable attractor in each growth condition, and the absence of oscillations in the normal range of operation;

- A parametrization of the models consistent with that of the previous models and with available literature data;

The resulting models include 13 variables and require the specification of 44 or 49 inequality constraints, for the gluconeogenic and glycolytic case, respectively (see Appendix B for the fully specified models). The guiding principles listed above were mostly sufficient for determining the inequality constraints between parameters, but in some situations several alternatives remained. In these cases we made an educated guess and chose one specific parametrization. In Sec. 3.5 we investigate the robustness of the modeling results for variations in parameter constraints.

\subsection{Steady states of network during growth on glucose and acetate}

The steady-state concentrations of the enzymes and regulatory proteins involved in carbon assimilation, both during growth on glucose and acetate, can be predicted from the models $\mathcal{M}_{\text {glyco }}$ and $\mathcal{M}_{\text {neo }}$, by means of the computer tool GNA (Sec. 2.3). Both $\mathcal{M}_{\text {glyco }}$ and $\mathcal{M}_{\text {neo }}$ have a single stable steady state. Given that we focus on qualitative properties of the system, we are particularly interested in the difference in steady-state concentrations during glycolysis and gluconeogenesis. That is, which genes are higher or lower expressed during growth on acetate as compared to growth on glucose? Table 1 summarizes the results of the comparison of the glycolytic and gluconeogenic steady states. For instance, the table shows that during growth on acetate, instead of glucose, the expression of the gene $p p s A$ increases whereas that of $p y k F$ decreases.

Do these predictions correspond to the available experimental data? Several data sets can be used to verify which genes are up-regulated or down-regulated when the growth conditions change. Oh et al. (2002) monitored transcription of $E$. coli by means of DNA microarrays during steady-state growth on acetate, using growth on glucose as a reference. Liu et al. (2005) also measured the transcription profiles by means of DNA microarrays, for six different carbon sources including glucose and acetate. Table 1 shows the relative expression changes for the genes that are included in the models $\mathcal{M}_{\text {glyco }}$ and $\mathcal{M}_{\text {neo }}$. As can be seen, the sign of the changes in expression is consistent between the two data sets, bearing in mind the experimental uncertainty. The experiment of Peng and Shimizu (2003) provided direct measurements of the accumulation of the enzymes in central carbon metabolism using 2-dimensional electrophoresis, during growth on different carbon sources, including glucose and acetate. The observed differences 
for the two enzymes included in our model (PykF and PpsA) are consistent with the measured transcription profiles of the corresponding genes.

The predicted changes in expression thus correspond well to the observations. The observed inversion of $p y k F$ and ppsA expression is correctly reproduced, as well as the down-regulation of gapA during growth on acetate. A switch in the expression of $p y k F$ and $p p s A$ is reported as a major signature of the glycolysis-gluconeogenesis transition, as it promotes the inversion of metabolic fluxes and the consumption of the new carbon source. The exception is the observed lower expression level of Crp in acetate-grown bacteria. This result is somewhat surprising as other measurements obtained under glucose depletion show the contrary (Ishizuka et al., 1993).

Is the correspondence with experimental data preserved when the indirect interactions are omitted from the model? In order to answer this question, we first computed the steady states of $\mathcal{M}^{0}$, the model accounting for purely transcriptional interactions (Appendix $\left[\right.$ ). Table 1 shows that $\mathcal{M}^{0}$ fails to reproduce most of the observed changes in gene expression. Indeed, $\mathcal{M}^{0}$ has a single steady state, so that the enzyme concentrations (e.g., PykF and PpsA) remain the same when the carbon source changes. Clearly, metabolic coupling is essential for the adjustment of gene expression when cells are alternatively grown on glucose and acetate.

But which mechanism is mostly responsible for the observed changes in gene expression? Are all indirect interactions equally important for the system response? Further insights into the role of indirect interactions can be gained from the analysis of reference models in which one coupling species (either Crp.cAMP or free FruR) is removed at a time (see Appendix $\mathrm{C}$ for the models). The results show that the control of the gene expression response is shared between different mechanisms, in the sense that the absence of a coupling species does not allow the experimental data to be completely reproduced (Table 1).

Crp.cAMP and free FruR affect the predicted adaptation differently, though. The control exerted via the Crp·cAMP complex equally affects the glycolytic enzymes and the global regulator fis, thus showing that Crp.cAMP-mediated coupling is at the heart of the regulatory system Nanchen et al. (2008). Moreover, removal of Crp·cAMP modifies the structure of the phase space itself, in the sense that a second steady state appears for $\mathcal{M}_{n e o / \text { Crp.cAmP }}^{0}$ which causes an ambiguity in the predicted expression levels of the glycolytic enzymes during gluconeogenesis. Metabolic coupling through free FruR deals with the control of metabolic fluxes, via the expression of glycolytic enzymes, but the expression of global regulators is not affected (Gutierrez-Ríos et al., 2007; Nanchen et al., 2008; Sarkar et al., 2008). 


\begin{tabular}{|c|c|c|c|c|c|c|c|c|}
\hline & crp & fis & $\operatorname{rpoS}$ & fruR & gapA & $p p s A$ & $p y k F$ & Reference vs model \\
\hline \multirow{3}{*}{$\begin{array}{l}\text { Experimental } \\
\text { data }\end{array}$} & $?$ & - & + & $?$ & \multirow[t]{3}{*}{-} & + & - & Oh et al. (2002) \\
\hline & - & - & + & + & & + & - & Liu et al. (2005) \\
\hline & & & & & & + & - & Peng and Shimizu (2003) \\
\hline \multirow{4}{*}{$\begin{array}{l}\text { Model } \\
\text { predictions }\end{array}$} & + & - & + & 0 & - & + & - & \multirow{4}{*}{$\begin{array}{rr}\mathcal{M}_{\text {neo }} \text { vs } \mathcal{M}_{\text {glyco }} \\
\mathcal{M}_{\text {neo } / \text { Crp·cAMP }}^{0} \text { vs } \mathcal{M}_{\text {glyco/Crp·cAMP }}^{0} \\
\mathcal{M}_{\text {neo /free FruR }}^{0} \text { vs } \mathcal{M}_{\text {glyco/free FruR }}^{0} \mathcal{M}^{0}\end{array}$} \\
\hline & 0 & 0 & + & 0 & $-/ 0$ & $+/ 0$ & $-/ 0$ & \\
\hline & + & - & + & 0 & + & 0 & 0 & \\
\hline & 0 & 0 & 0 & 0 & 0 & 0 & 0 & \\
\hline
\end{tabular}

Table 1: Predicted and observed differences in gene expression during growth on acetate vs growth on glucose. The measurements concern the differences in mRNA concentrations (Liu et al., 2005; Oh et al., 2002) and protein concentrations (Peng and Shimizu, 2003). The model predictions are the differences in steady-state concentrations (+: higher, - : lower, 0: equal). In some cases the precision of the measurements does not allow to distinguish between qualitative differences, indicated by ?. If the model predictions agree (disagree) with the data, the entry is colored green (red). If the data are ambiguous, the predicted entry is shown in yellow. $\mathcal{M}_{n e o / C r p \cdot c A M P}^{0}$ has two steady states, leading to ambiguous predictions.

\begin{tabular}{l|cccccccl} 
& crp & fis & rpoS & fruR & gapA & ppsA & pykF & Reference/Model \\
\hline Experimental data & - & - & - & 0 & - & + & 0 & Kao et al. (2005) \\
\hline \hline Model & + & - & - & 0 & - & + & 0 & $\mathcal{M}_{\text {glyco }}$ \\
predictions & 0 & 0 & 0 & - & - & + & - & $\mathcal{M}_{\text {glyco/Crp.cAMP }}^{0}$ \\
& 0 & 0 & 0 & 0 & 0 & 0 & 0 & $\mathcal{M}_{\text {glyco/free FruR }}^{0}$ \\
& 0 & 0 & 0 & 0 & 0 & 0 & 0 & $\mathcal{M}^{0}$ \\
\hline
\end{tabular}

Table 2: Predicted and observed initial responses on the transcriptional level when glucose is exhausted. The model predictions are the immediate change in the protein synthesis rate following the exhaustion of glucose, in comparison with the steady-state protein synthesis rate during glycolysis ( + : higher, - : lower, 0 : equal). The measurements concern the initial change in mRNA concentrations when the bacteria are transferred from a glucose to acetate medium (Kao et al., 2005). See Table1 for the color codes. 


\subsection{Dynamic response of gene expression to diauxic shift}

When glucose is exhausted the bacteria are able to continue growth on an alternative carbon source like acetate. Such a diauxic shift entails important changes in metabolism, as well as a reorganization of gene expression. The dynamic response of gene expression has two aspects: an initial response on the transcriptional level when the glucose-grown bacteria sense the rapid diminution of substrate levels, and the eventual response on the enzyme level after the bacteria have switched to the utilization of acetate. Both of these responses can be taken into account using the $\mathcal{M}_{\text {glyco }}$ and $\mathcal{M}_{\text {neo }}$ models. In the former case, we consider the steady state of the glycolytic model and predict the immediate change of the protein synthesis rates during glucose depletion, bearing in mind that the protein synthesis rates are proportional to the mRNA concentrations (Kremling, 2007). In the latter case, we follow the predicted changes in enzyme levels in the gluconeogenic model, starting from an initial state that corresponds to the steady state in glycolysis. We make the assumption that on the time-scale of the dynamics of protein concentrations, which is on the order of hours in $E$. coli, the changes in metabolic fluxes are rapid. This assumption is supported by the available experimental data (Hardiman et al., 2007).

The predicted initial response of the bacteria to glucose depletion is shown in Table 2. We compare the synthesis rate of the proteins in the glycolysis steady state and in the successor state following the rapid drop in glucose levels (which is modeled in $\mathcal{M}_{\text {glyco }}$ by setting the glucose concentration variable below its threshold, see Appendix A). The synthesis rates of most proteins are seen to react immediately to the change in nutrient availability. In fact, the sharp increase of cAMP activates Crp which has a direct effect on many of the targets of this protein, which in our model consist of fis, cya, gyrI, gapA, rpoS, and crp itself 11 These predictions can be compared with the experimental data of Kao et al. (2005), who used DNA microarrays to measure the transcriptional response of cells grown on glucose to mid-exponential phase and then reinoculated in a medium containing acetate. As can be seen, the predicted signs of the change in mRNA concentrations agree with the signs computed from the data.

The above adaptation of the bacterial cells occurs minutes after the change of growth conditions. In order to assess the response on the time-scale of protein synthesis and degradation, that is, hours after the change in carbon source, we perform a qualitative simulation with the $\mathcal{M}_{\text {neo }}$ model, taking the glycolysis steady state as our initial state. The system is seen to converge to the gluconeogenic steady state, in the sense that all paths in the state transition graph eventually reach this state. The different paths represent possible sequences of qualitative events leading the system to the new steady state (see Fig. 66 for an example). We exploited model-checking techniques to characterize properties common to all paths. Given that the state transition graph is generated from a specified ordering of parameter values, rather than exact numerical values, such properties are robust with respect to small quantitative changes in parameter values and capture fundamental aspects of the underlying regulatory structure of the network.

\footnotetext{
${ }^{1}$ Notice that the inhibitory effect of CRP.cAMP complex on the transcription of RpoS remains somewhat controversial (see Mandel and Silhavy (2005); Venturi (2003); Zgurskava et al. (1997) and references therein).
} 


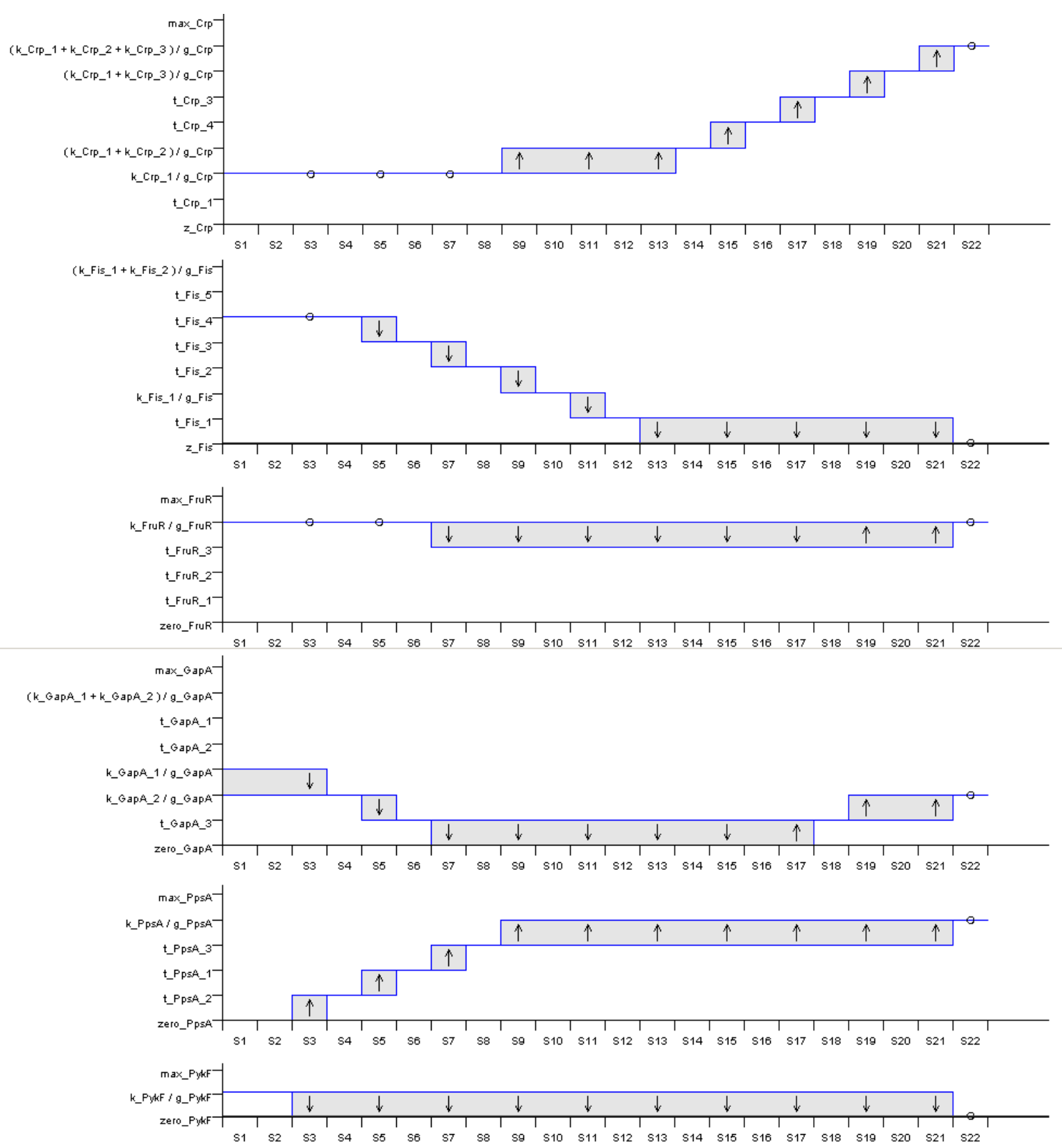

Figure 6: Example of a qualitative simulation of the glucose-acetate diauxie (Sec. 2.3). The selected pathway shows the typical dynamics of protein concentrations following the shift to acetate. The glycolytic enzymes (PpsA, GapA, PykF) are the first to respond. Global regulators (Crp, Fis, FruR) respond later, once a sufficiently high level of PpsA is reached. The path is a sequence of qualitative states, in each of which the concentration variables have a specific derivative sign (steady, o; increasing, $\uparrow$; decreasing, $\downarrow$ ). Transitions between qualitative states correspond to qualitative events, notably threshold crossings of the variables.

RR $n^{\circ} 7617$ 
Following the shift to acetate, the glycolytic enzymes are the first to respond. The level of PpsA, essential for the metabolism of gluconeogenic substrates, starts to increase immediately after the diauxie, whereas PykF and GapA are progressively down-regulated, due to the high levels of free FruR in the new growth conditions. The expression of the genes encoding the global regulators responds later. A sufficiently high level of PpsA is required for the accumulation of Crp.cAMP, and the change in expression of the genes fis and crp, which are under the control of this regulator.

Interestingly, the positive influence of PpsA on the accumulation of Crp·cAMP in the absence of glucose, an indirect interaction inferred by the method described in Sec. 2.1, is confirmed by the experimental work of Kao et al. (2005). They show that in a ppsA mutant the bacteria took much longer to resume growth on acetate after the exhaustion of glucose. The wild-type phenotype could be partially rescued by the addition of cAMP, which suggests that PpsA controls the concentrations of cAMP and Crp·cAMP, as predicted by the model.

The importance of metabolic coupling can be assessed by comparing the above predictions of the response of the system to the exhaustion of glucose with the predictions obtained without indirect interactions $\left(\mathcal{M}^{0}\right)$. In the latter case the network does not respond to the depletion of glucose, in the sense that the steady states for growth on glucose and growth on acetate are identical (Sec. 3.3). Indeed, in the absence of metabolic coupling the transcription factors Crp and FruR cannot sense the change in concentration of their metabolic effectors, cAMP and FBP, which plays a major role in the initiation of the system response. For instance, analysis of model $\mathcal{M}_{\text {glyco }}^{0}$ free FruR shows that the removal of free FruR-mediated coupling completely blocks the system response.

\subsection{Robustness analysis to parameter changes}

As explained in Sec. 3.2 , the qualitative PL models involve choices for inequality constraints that are underdetermined by the available experimental data. The indirect interactions due to metabolic coupling involve parameters that characterize lumped processes occurring on different time-scales, so that a direct measure of their strength is difficult to obtain experimentally. The question can be asked, first, to which extent the results obtained with the models $\mathcal{M}_{\text {glyco }}$ and $\mathcal{M}_{n e o}$ are robust to changes in the parameter constraints. Second, the indirect interactions are not expected to all have the same importance, so it would be interesting to identify those interactions for which a specific ordering between parameters is critical for reproducing the observed behavior of the network.

Within the criteria introduced in Sec. 3.2, we analyzed the robustness of our results by randomly changing the ordering of thresholds characterizing the indirect interactions mediated by either Crp·cAMP or free FruR. Each different ordering leads to a different set of inequality constraints, and thus to a different qualitative PL model (see Appendix D for details on the alternative sets of inequality constraints). For each of the above-mentioned models, the gene expression response during a glucose-acetate diauxie is analyzed, in the way described in Sec. 3.3 and Sec. 3.4, and compared to the prediction of the original models $\mathcal{M}_{\text {glyco }}$ and $\mathcal{M}_{\text {neo }}$. For each gene in the network, we define a robustness index as the fraction of models for which the predictions remain stable after a change of inequality constraints (see Chaves et al. (2006) for another approach to test the robustness of predictions of qualitative models). 
The results of this robustness analysis are shown in Table 3, for the predictions of steady-state and dynamic responses. Overall, the model predictions are robust to changes in parameter constraints, thus emphasizing the importance of the network topology for the emergence of the observed response. The major exception are variations in the parameter constraints for free FruR, which may strongly affect affect the predictions of enzyme steady-state levels. This could be explained by the fact that the space of possible parameter orderings for free FruR is much less constrained by the available biological data than that for Crp·cAMP. We find that a fine-tuning of the mutual regulation between gap $A$ and $p p s A$, via metabolic coupling, is essential for the emergence of a correct system response.

\begin{tabular}{c|ccccccc} 
& crp & fis & rpoS & fruR & gapA & ppsA & pykF \\
\hline Crp·cAMP (20) & 0.85 & 1 & 1 & 0.35 & 1 & 1 & 1 \\
\hline Free FruR (15) & 0.07 & 1 & 1 & 0.67 & 0.07 & 0.07 & 0.07 \\
\hline Total (35) & 0.51 & 1 & 1 & 0.49 & 0.6 & 0.6 & 0.6 \\
\hline & $c r p$ & fis & rpoS & fruR & gapA & ppsA & pykF \\
\hline Crp·cAMP (20) & 0.6 & 0.6 & 0.6 & 1 & 1 & 1 & 1 \\
\hline free FruR (20) & 1 & 1 & 1 & 0.8 & 0.2 & 0.5 & 0.5 \\
\hline Total (40) & 0.8 & 0.8 & 0.8 & 0.9 & 0.6 & 0.75 & 0.75 \\
\hline
\end{tabular}

Table 3: Robustness index of $A$ : steady-state predictions (Table 1) and $B$ : initial transcriptional response (Table 2), when changing the relative strength of indirect interactions in models $\mathcal{M}_{\text {glyco }}$ and $\mathcal{M}_{\text {neo }}$. The number of alternative parameter orderings, derived from $\mathcal{M}_{n e o}$, are indicated between parentheses.

\section{Conclusions}

Metabolic coupling gives rise to indirect interactions between enzymes and genes in gene regulatory networks, which raises the question how important these interactions are for the network dynamics. In particular, we asked if metabolic coupling is essential for shaping the response of the cell or if it has a minor influence. In order to address this issue, we have used simple, qualitative models for exploring the qualitative network dynamics and analyzed by means of these models the gene regulatory network controlling carbon assimilation in E. coli.

The philosophy of our approach differs from other work studying the interactions between metabolism and gene regulation. One line of research starts with fully specified kinetic models of integrated networks of enzymatic reactions and protein synthesis and degradation reactions (e.g., Klipp et al. (2005); Kotte et al. (2010) ). In such models, which provide a fine-grained mechanistic picture, metabolic coupling can be seen to emerge from the underlying reaction network. Changes in gene expression modify the metabolic flux distribution, which leads to changes in metabolite concentrations and thus affects the activity of transcription factors and other regulatory proteins. Another type of approach consists of the various extensions of flux balance analysis (FBA) that aim at integrating gene regulation with metabolism (e.g., Covert et al. (2004, 2008); Shlomi et al. (2007)). These methods sidestep the explicit specification 
of rate laws, by making a quasi-steady-state assumption, generating the flux distribution that satisfies an objective function, for instance optimal biomass production, and modeling gene regulation by Boolean rules. This enables the prediction of changes in the metabolic flux distribution over time, under different experimental conditions, and the effect of these changes on the activity of transcription factors. Our approach differs from the two alternatives mentioned above in that we do not explicitly include metabolism in our model nor predict flux distributions and metabolite concentrations. Rather, we identify $a$ priori the indirect interactions mediated by metabolism that couple the expression levels of genes coding for enzymes to those encoding transcription factors. Our approach can thus be seen as a model reduction that uncovers the effective network structure on the time-scale of gene expression.

The results of the application of our approach to the $E$. coli network show that indirect interactions completely modify the predicted expression patterns following a glucose-acetate shift, transforming metabolic genes from passive to active players of the adaptive response of the cell. The profiles obtained when including metabolic coupling show good correspondence with the available experimental data on the steady-state and dynamic response, contrary to what is observed when the model is restricted to transcriptional regulation only. In particular, key signatures of growth on a glycolytic substrate (glucose) as compared to growth on a neoglucogenic substrate (acetate) are reproduced, such as the opposite expression levels of the enzymes PpsA and PykF. The indirect interactions arising from metabolic coupling are found to be crucial, because they account for rapid changes in metabolism (fluxes, metabolite concentrations) that control the activity of transcriptional regulators. This allows environmental perturbations, such as the depletion of glucose, to propagate through the network and affect the expression of a large number of genes. While these results have been obtained for one particular bacterial model system, the ubiquity of metabolic coupling in regulatory networks suggests that our conclusions are relevant for other systems as well.

\section{Acknowledgments}

This work was supported by the European Commission under project ECMOAN (FP6-2005-NEST-PATH-COM/043235) and the Agence Nationale de la Recherche under project MetaGenoReg (ANR-06-BYOS-0003). 


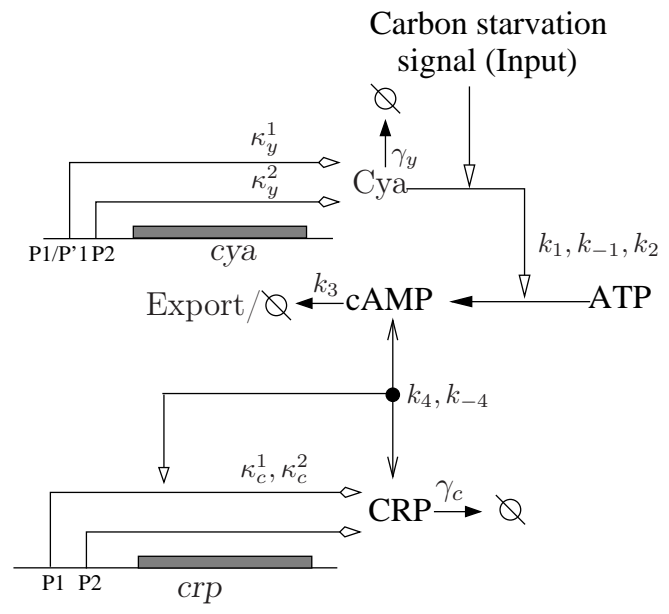

Figure 7: Crp activation network.

\section{A Kinetic model of subnetwork controlling Crp activation}

The CRP activation subnetwork is composed of the proteins Cya, CRP, and the complex CRP·cAMP (see Fig. 7). When glucose runs out, Cya is activated through the phosphotransferase system (PTS), and catalyzes the conversion of ATP into cAMP (Saier et al., 1996). For simplicity, we do not describe the phosphotransferase system in our model, but rather consider a carbon starvation signal that directly stimulates the activity of Cya, and thus the synthesis of cAMP. This signal is identified with the glucose concentration.

\begin{tabular}{cc|cc} 
Parameter & Value & Parameter & Value \\
\hline \hline$\kappa_{y}^{1}$ & $2.6 \cdot 10^{-13} M^{-1} s^{-1}$ & $\kappa_{y}^{2}$ & $2.27 \cdot 10^{-13} M^{-1} s^{-1}$ \\
$\kappa_{c}^{1}$ & $6.89 \cdot 10^{-9} M^{-1} s^{-1}$ & $\kappa_{c}^{2}$ & $5.75 \cdot 10^{-8} M^{-1} s^{-1}$ \\
$k_{1}$ & $1.3210^{8} M^{-1} s^{-1}$ & $k_{-1}$ & $0.64 s^{-1}$ \\
$k_{2}$ & $135 s^{-1}$ & $k_{3}$ & $1.007 s^{-1}$ \\
$k_{4}$ & $8.28 \cdot 10^{5} M^{-1} s^{-1}$ & $k_{-4}$ & $0.74 s^{-1}$ \\
$\gamma_{y}$ & $0.0062 s^{-1}$ & $\gamma_{c}$ & $0.303 s^{-1}$ \\
$\theta_{\text {Crp } \cdot \text { cAMP }}^{1}$ & $8.27 \cdot 10^{-6} \mathrm{M}$ & $\theta_{\text {Crp.cAMP }}^{2}$ & $3.46 \cdot 10^{-7} \mathrm{M}$ \\
\hline
\end{tabular}

Table 4: Parameter values for the kinetic model of the subnetwork controlling Crp activation (Ropers et al., 2011).

In Ropers et al. (2011) we used standard approaches of biochemistry to develop a detailed kinetic model of the carbon activation network : 


$$
\begin{aligned}
& \dot{x}_{\text {Cya free }}=\kappa_{y}^{1}+\kappa_{y}^{2} h^{+}\left(x_{C r p \cdot c A M P}, \theta_{\text {Crp.cAMP }}^{2}, m_{C r p \cdot c A M P}\right)-\gamma_{y} x_{\text {Cya free }} \\
& +\left(k_{-1}+k_{2} h^{+}\left(u_{G l c}, \theta_{G l c}, m_{G l c}\right)\right) x_{C y a \cdot A T P}-k_{1} x_{C y a} \text { free } u_{A T P} \\
& \dot{x}_{\text {Crp free }}=\kappa_{c}^{1}+\kappa_{c}^{2} h^{+}\left(x_{C r p \cdot c A M P}, \theta_{\text {Crp.cAMP }}^{1}, m_{C r p \cdot c A M P}\right)-\gamma_{c} x_{C r p \text { free }} \\
& +k_{-4} x_{C r p . c A M P}-k_{4} x_{C r p \text { free }} x_{c A M P} \text { free } \\
& \dot{x}_{\text {Cya } \cdot A T P}=k_{1} x_{\text {Cya free }} u_{A T P}-\left(k_{-1}+k_{2} h^{+}\left(u_{G l c}, \theta_{\text {Glc }}, m_{\text {Glc }}\right)+\gamma_{y}\right) x_{\text {Cya ATP }} \\
& \dot{x}_{\text {Crp.cAMP }}=k_{4} x_{C r p \text { free }} x_{c A M P \text { free }}-\left(k_{-4}+\gamma_{c}\right) x_{C r p \cdot c A M P} \\
& \dot{x}_{c A M P \text { free }}=k_{2} h^{+}\left(u_{G l c}, \theta_{G l c}, m_{G l c}\right) x_{C y a \cdot A T P}+k_{-4} x_{C r p \cdot c A M P}-k_{3} x_{c A M P \text { free }} \\
& -k_{4} x_{C r p \text { free }} x_{c A M P} \text { free }
\end{aligned}
$$

where $x_{C y a f r e e}, x_{C r p \text { free }}$, and $x_{C A M P \text { free }}$ denote the concentrations of free (unbound) Cya, Crp, and cAMP, respectively, and $x_{C y a \cdot A T P}$ and $x_{C r p \cdot c A M P}$ denote the concentrations of the complexes Cya.ATP and Crp.cAMP, respectively. $u_{G l c}$ and $u_{A T P}$ denote the glucose and ATP concentrations. The kinetic constants are associated with the reaction rates in Fig. 7. The above model includes fast and slow reactions, with high and low reactions rates, respectively (see Table 4 for parameter values). Typically, complex formation and enzymatic reactions are fast, while protein synthesis and degradation are slow. Based on this distinction, we define slow variables (typically, total protein concentrations) and fast variables (concentrations of biochemical complexes and metabolites) as linear combinations of the original variables:

$$
\begin{aligned}
x_{C y a} & =x_{\text {Cya free }}+x_{C y a \cdot A T P} \\
x_{C r p} & =x_{C r p \text { free }}+x_{C r p \cdot c A M P} \\
x_{c A M P} & =x_{c A M P \text { free }}+x_{C r p \cdot c A M P}
\end{aligned}
$$

The variable transformation ensures that slow variables change through slow reactions only, while fast reactions dominate the dynamics of the fast variables. With the above transformation, the system of Eq. 10 can be rewritten as:

$$
\begin{aligned}
& \text { Slow system } \\
\dot{x}_{C y a}= & \kappa_{y}^{1}+\kappa_{y}^{2} h^{+}\left(x_{C r p \cdot c A M P}, \theta_{\text {Crp } \cdot c A M P}^{2}, m_{\text {Crp } \cdot c A M P}^{2}\right)-\gamma_{y} x_{C y a} \\
\dot{x}_{C r P}= & \kappa_{c}^{1}+\kappa_{c}^{2} h^{+}\left(x_{C r p \cdot c A M P}, \theta_{\text {Crp } \cdot c A M P}^{1}, m_{\text {Crp } \cdot c A M P}^{1}\right)-\gamma_{c} x_{C r p} \\
& \text { Fast system } \\
\dot{x}_{C y a \cdot A T P}= & k_{1}\left(x_{C y a}-x_{C y a \cdot A T P}\right) u_{A T P}- \\
& \left(k_{-1}+k_{2} h^{+}\left(u_{G l c}, \theta_{G l c}, m_{G l c}\right)+\gamma_{y}\right) x_{C y a \cdot A T P} \\
\dot{x}_{C r p \cdot c A M P}= & k_{4}\left(x_{C r p}-x_{C r p \cdot c A M P}\right)\left(x_{c A M P}-x_{C r p \cdot c A M P}\right)- \\
& \left(k_{-4}+\gamma_{c}\right) x_{C r p \cdot c A M P} \\
\dot{x}_{c A M P}= & k_{2} h^{+}\left(u_{G l c}, \theta_{G l c}, m_{G l c}\right) x_{C y a \cdot A T P}+\left(k_{3}-\gamma_{C r p}\right) x_{C r p \cdot c A M P}- \\
& k_{3} x_{C A M P}
\end{aligned}
$$


We apply the quasi-steady state (QSS) assumption to the reformulated system, by putting the time derivative of fast variables equal to 0 and neglecting the contribution of the slow variables to the dynamics of the fast variables (Heinrich and Schuster, 1996). This results in a system of algebraic equations for the fast variables that can be explicitly solved, leading to the following reduced system at the slow time-scale:

$$
\begin{aligned}
\dot{x}_{C y a} & =\kappa_{y}^{1}+\kappa_{y}^{2}-\gamma_{y} x_{C y a} \\
\dot{x}_{C r p} & =\kappa_{c}^{1}+\kappa_{c}^{2} h^{+}\left(x_{C r p \cdot c A M P}, \theta_{C r p \cdot c A M P}^{1}, m_{C r p \cdot c A M P}\right)-\gamma_{c} x_{C r p} \\
x_{C r p \cdot c A M P} & =\frac{h^{+}\left(u_{G l c}, \theta_{G l c}, m_{G l c}\right) x_{C y a}}{h^{+}\left(u_{G l c}, \theta_{G l c}, m_{G l c}\right) x_{C y a}+K_{4} K_{3}} x_{C r p} \\
K_{3} & =\frac{k_{3}}{k_{2}} \\
K_{4} & =\frac{k_{-4}}{k_{4}}
\end{aligned}
$$

This model has been used to generate the plot in Fig. 3 .

\section{B PL models $\mathcal{M}_{\text {glyco }}$ and $\mathcal{M}_{\text {neo }}$}

The complete PL models for glycolysis and gluconeogenesis $\left(\mathcal{M}_{\text {glyco }}\right.$ and $\left.\mathcal{M}_{\text {neo }}\right)$ are reported in figures 8 and 9, respectively. Parameter inequalities are also specified. The models contain 13 variables and an external signal $\left(u_{G l c}\right)$, accounting for the presence of glucose in the medium. The latter is used in model $\mathcal{M}_{\text {glyco, }}$, when studying the fast response of the mRNA levels to a glucose depletion (see Sec. 3.4).

\section{PL models of $\mathcal{M}^{0}$ reference networks}

In order to better understand the role of indirect interactions in the emergence of the system response, we built a number of reference models in which all indirect interactions mediated by Crp.cAMP or free FruR are systematically removed. These models are called $\mathcal{M}_{\text {glyco/Crp } \cdot c A M P}^{0}, \mathcal{M}_{\text {neo/Crp cAMP }}^{0}, \mathcal{M}_{\text {glyco/free FruR }}^{0}$, $\mathcal{M}_{\text {neo free FruR }}^{0}$ and $\mathcal{M}^{0}$. They are obtained from the original models $\mathcal{M}_{\text {glyco }}$ and $\mathcal{M}_{\text {neo }}$ by removing the selected coupling species while keeping the effect of transcription factors (Sec. 3.3). Fig. 10 shows the resulting network topology

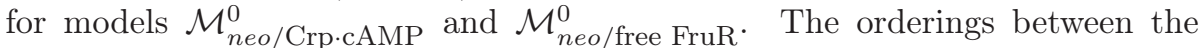
remaining threshold parameters, after elimination of indirect interactions, are preserved. State equations and parameter inequalities for all reference models are reported in Fig. 11 to Fig. 15. 


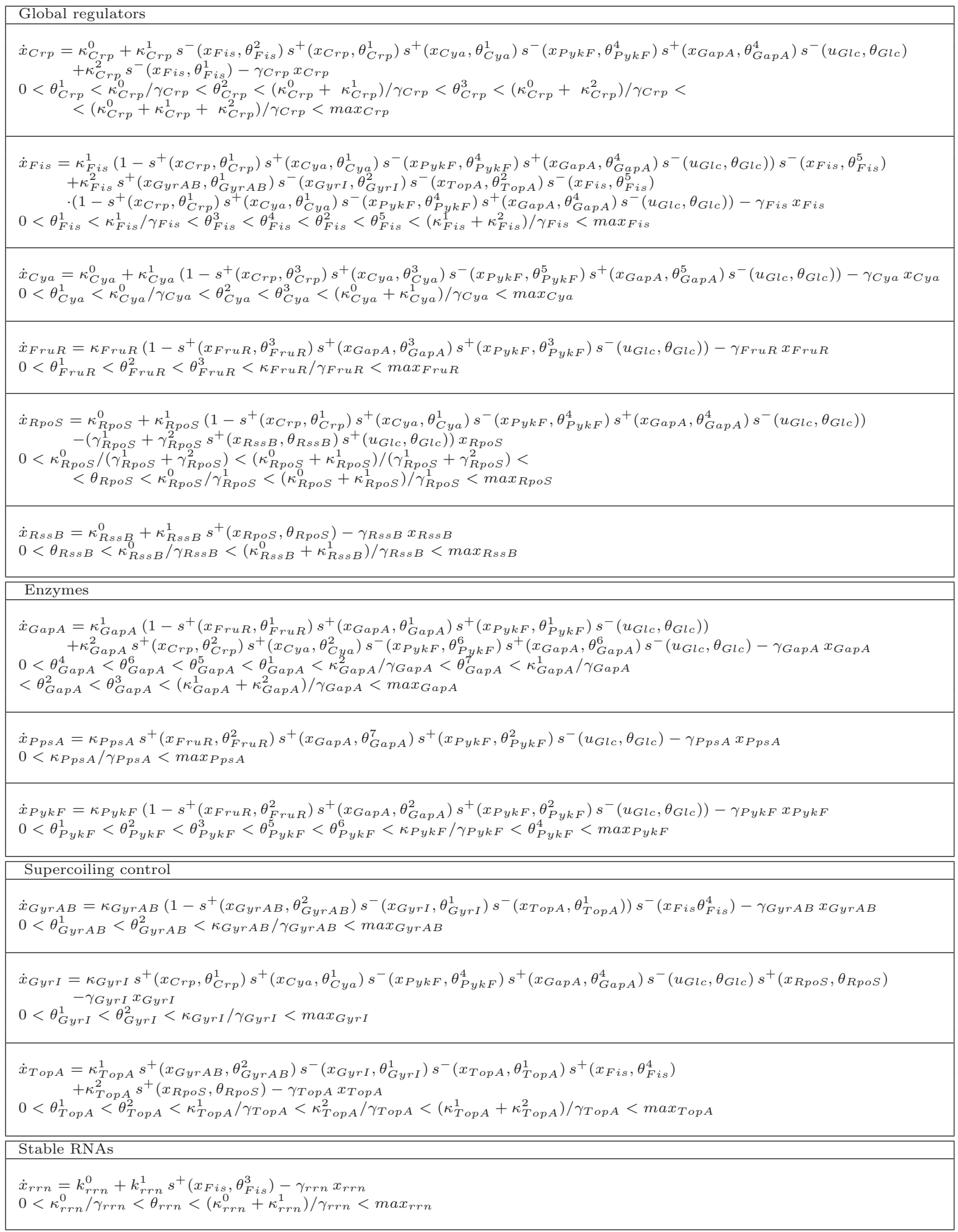

Figure 8: PL equations and parameters inequalities for model $\mathcal{M}_{\text {glyco }}$. 


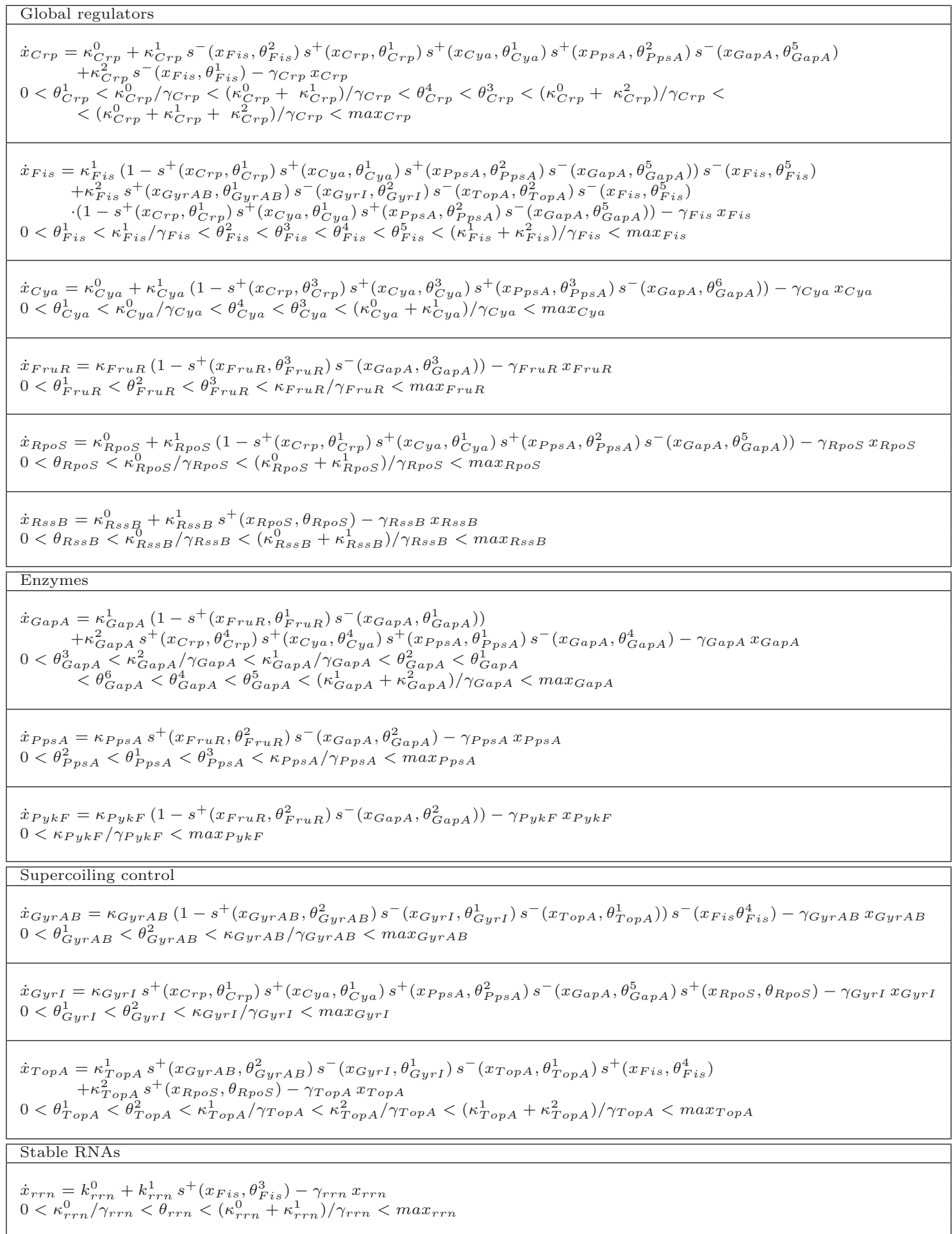

Figure 9: PL equations and parameters inequalities for model $\mathcal{M}_{\text {neo }}$. 


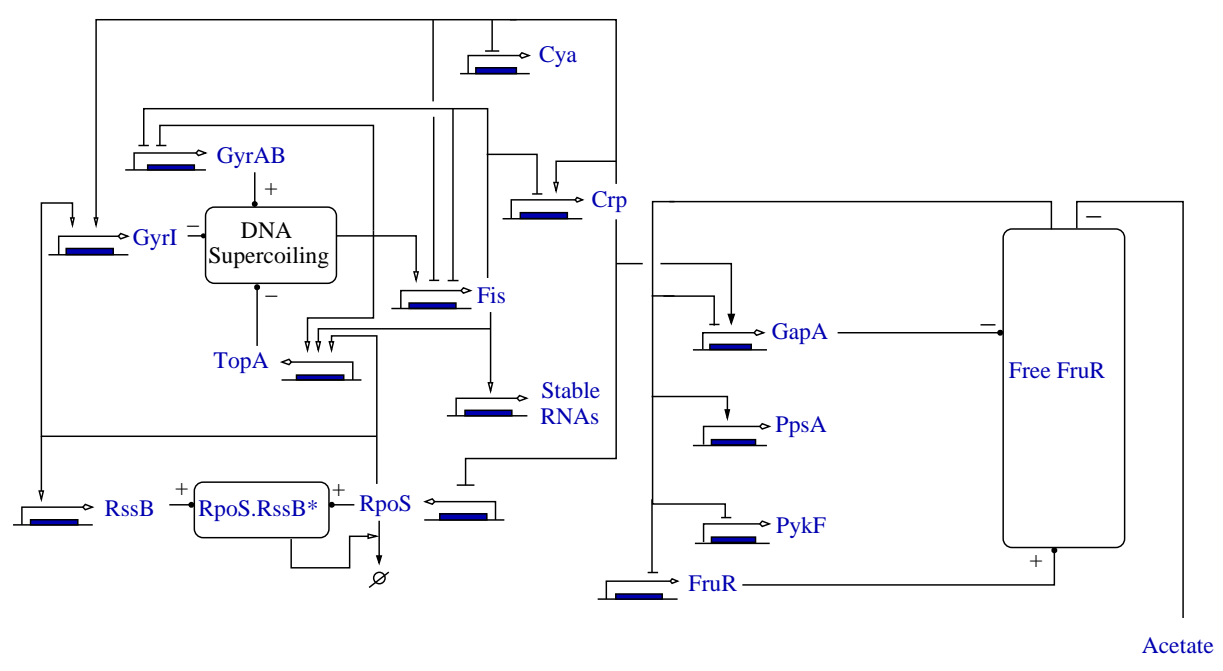

A

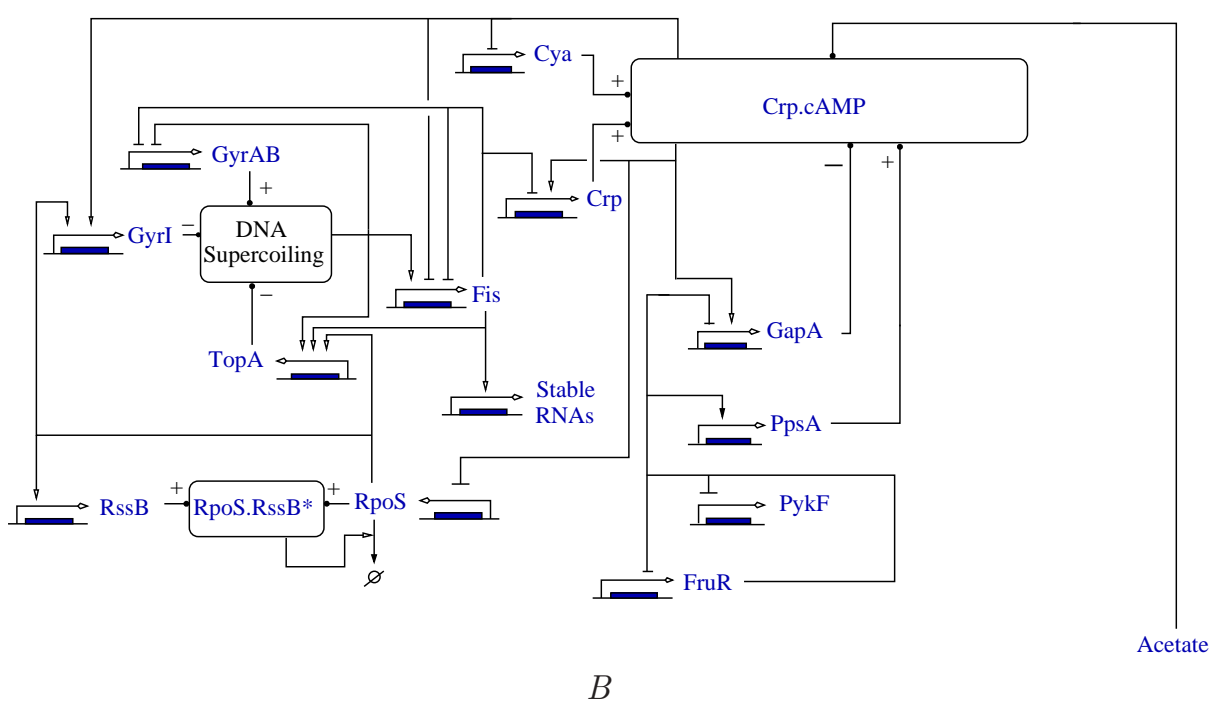

Figure 10: Regulatory networks $A: \mathcal{M}_{\text {neo/Crp.cAMP }}^{0}$ and $B: \mathcal{M}_{\text {neo/free FruR }}^{0}$, obtained by removing one coupling species from model $\mathcal{M}_{\text {neo }}$. 


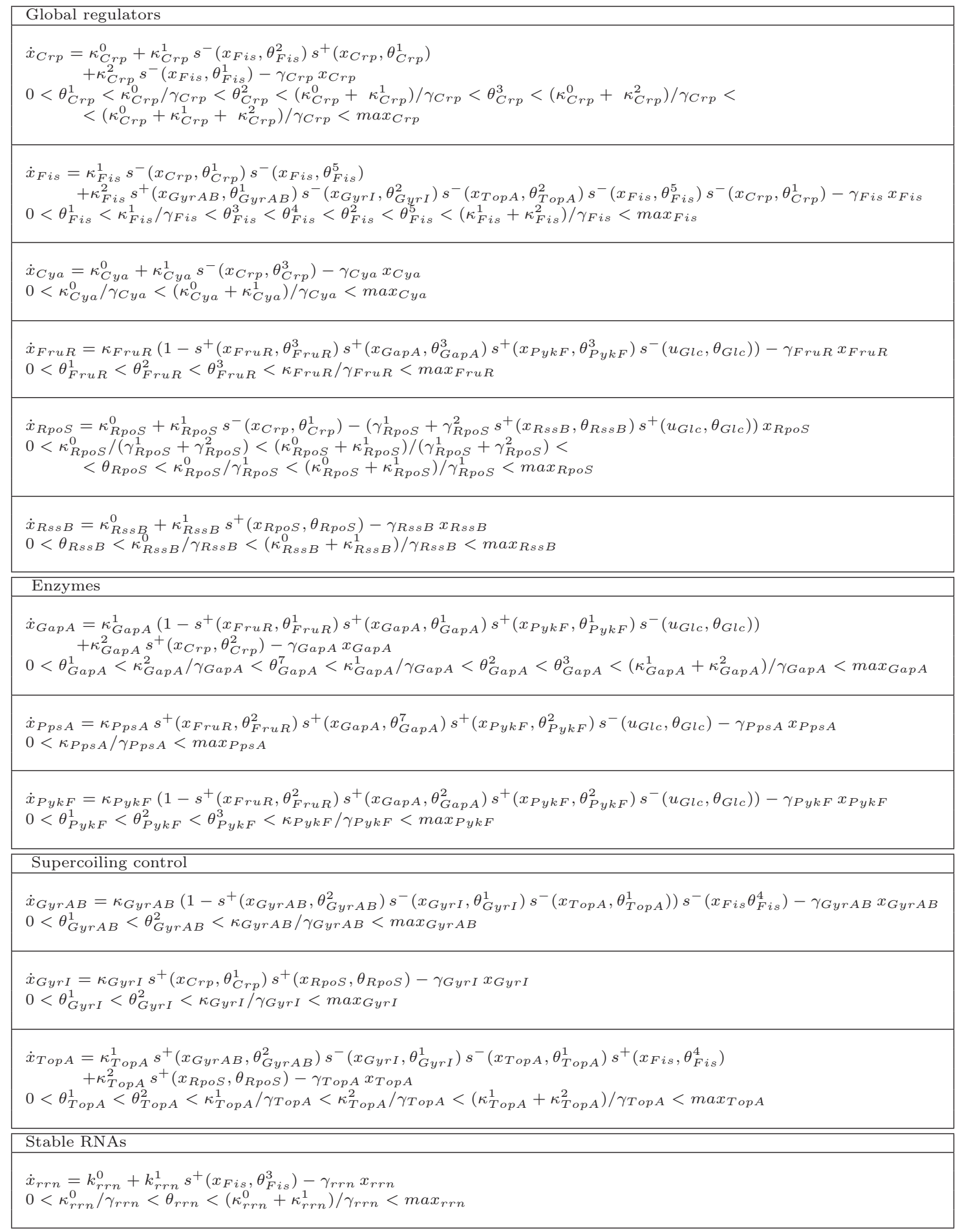

Figure 11: PL equations and parameter inequalities for model $\mathcal{M}_{\text {glyco/Crp cAMP }}^{0}$. 


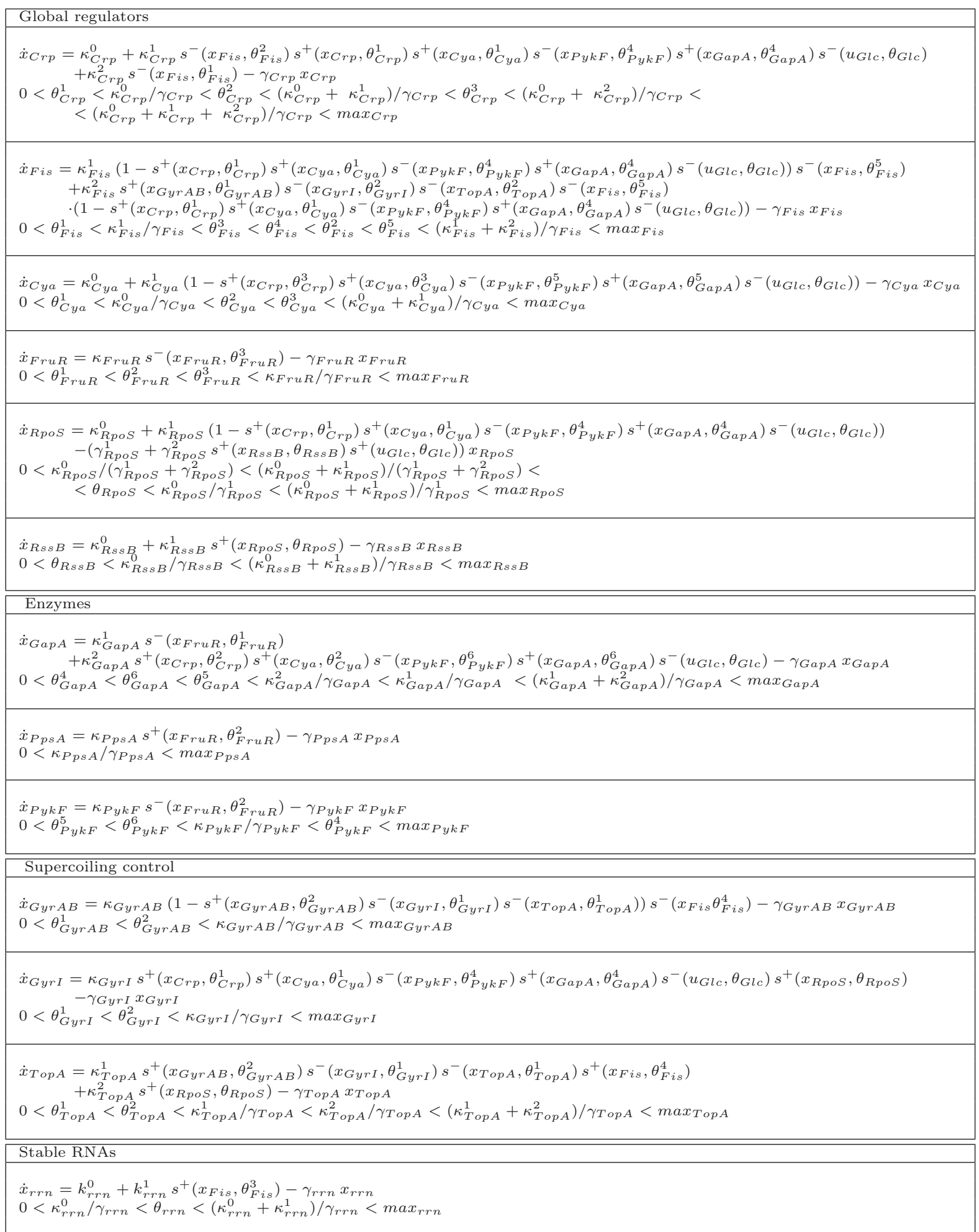

Figure 12: PL equations and parameter inequalities for model $\mathcal{M}_{\text {glyco/free FruR }}^{0}$. 


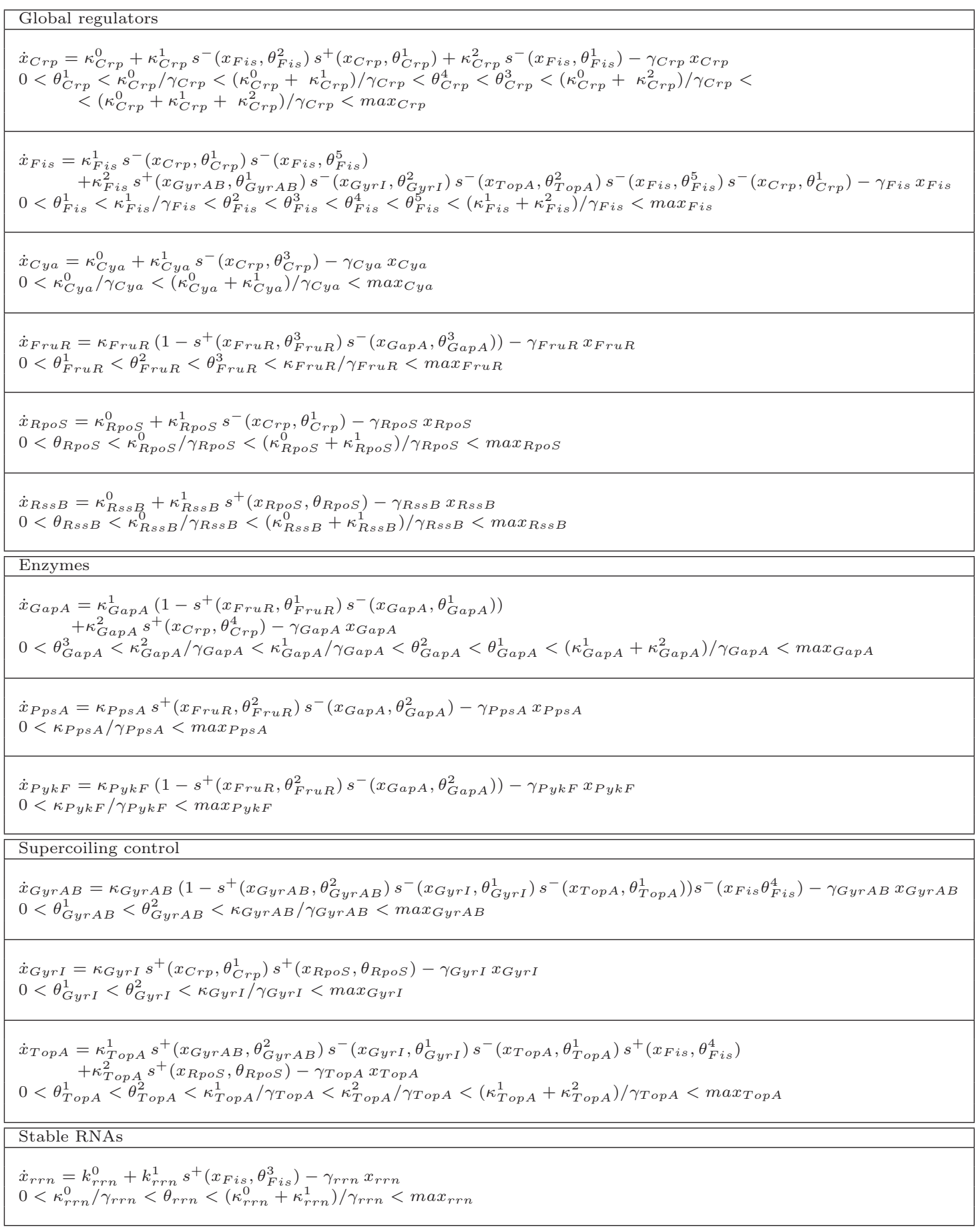

Figure 13: PL equations and parameter inequalities for model $\mathcal{M}_{\text {neo/Crp.cAMP }}^{0}$. 


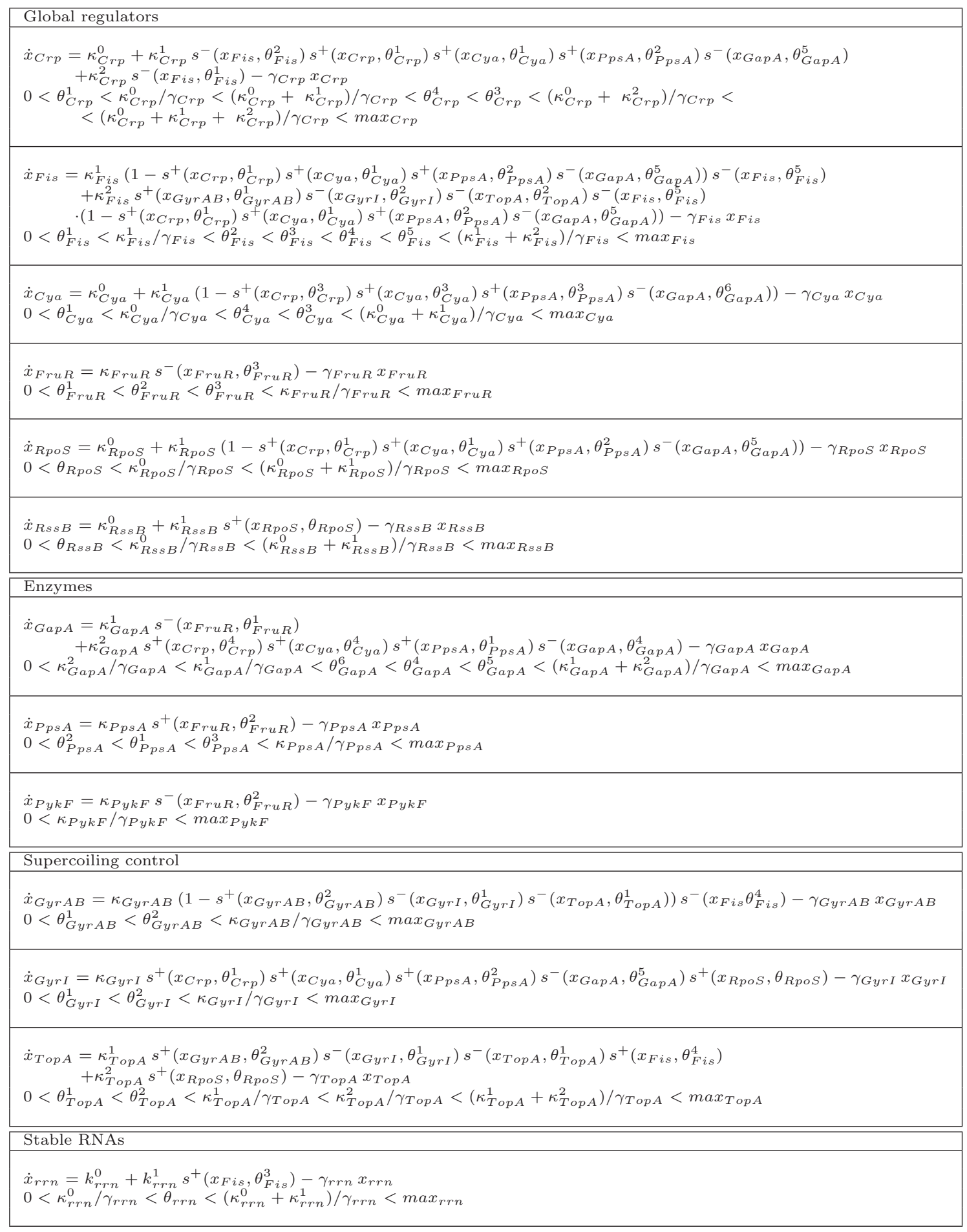

Figure 14: PL equations and parameter inequalities for model $\mathcal{M}_{\text {neo/free FruR }}^{0}$. 


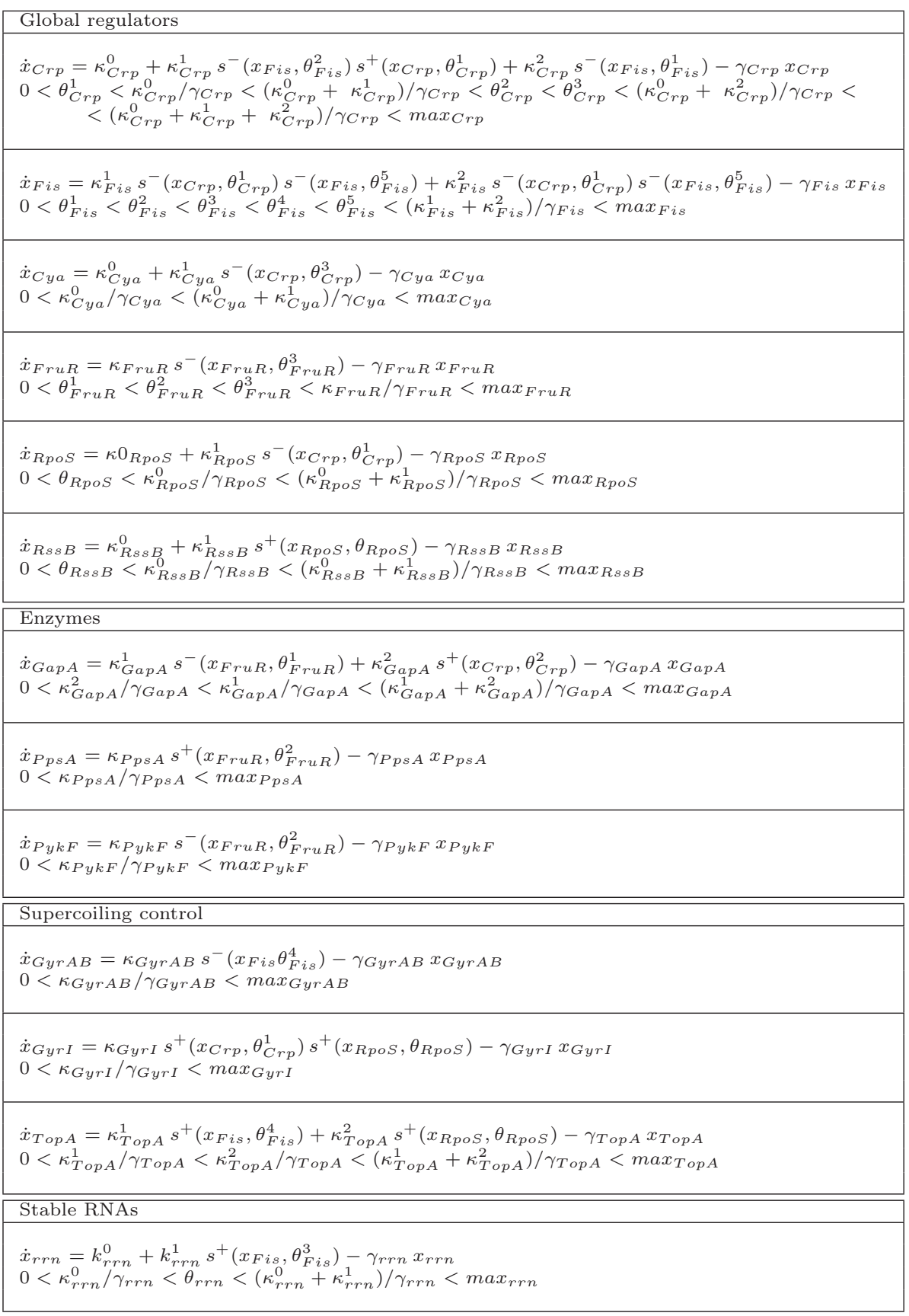

Figure 15: PL equations and parameter inequalities for model $\mathcal{M}^{0}$. This model is the same for both glycolysis and gluconeogenesis. 


\section{Model variants of $\mathcal{M}_{\text {glyco }}$ and $\mathcal{M}_{\text {neo }}$ used for ro- bustness tests}

In order to asses the robustness of the results obtained with the models $\mathcal{M}_{\text {glyco }}$ and $\mathcal{M}_{n e o}$, we built a set of model variants by randomly changing the ordering of thresholds characterizing the indirect interactions mediated by either Crp·cAMP or free FruR.

Each model variant is submitted to biological and mathematical constraints, reflecting current biological knowledge on the system and structural dependencies between variables. We illustrate the general principles on a small example representing indirect interactions mediated by free FruR during gluconeogenesis. The topology of this subnetwork and the corresponding PL model are shown in Fig. 16. The amount of the coupling species free FruR depends on the concentration of proteins FruR and GapA (which control the concentration of FBP). Free FruR regulates the expression of four target genes: fruR, gapA, ppsA, and $p y k F$. The corresponding PL model shown in the figure has six distinct thresholds: three for the concentration of FruR $\left(\theta_{F r u R}^{1}, \theta_{F r u R}^{2}, \theta_{F r u R}^{3}\right)$ and three for GapA $\left(\theta_{\text {Gap } A}^{1}, \theta_{\text {Gap A }}^{2}, \theta_{\text {GapA }}^{3}\right)$, where we assume that the control of $p y k F$ and pps $A$ occurs at the same concentration level. The difficulty consists in choosing a plausible ordering of these thresholds.

A first constraint on the threshold ordering derives from the definition of indirect interactions itself and tightly links the relative order of the $\theta_{\text {Gap } A}$ and the $\theta_{\text {FruR }}$ thresholds. We briefly explain why.

Similar to the example in Fig. 3 of the main text, the concentration of free FruR at steady state can be approximated by a function of the concentrations of FruR and GapA:

$$
x_{\text {FruR free }}=g\left(x_{\text {FruR }}, x_{\text {Gap } A}, u_{G l c}\right) \approx \frac{K}{x_{G a p A}+K} x_{F r u R}
$$

where $K$ is a lumped kinetic parameter. We plot $h^{+}\left(x_{\text {FruR free }}, \theta_{\text {FruR free }}\right)$ as a function of FruR and GapA in Fig. 17 for two different values of the threshold concentration $\left(\theta_{\text {FruR free }}^{1}\right.$ and $\left.\theta_{\text {FruR free }}^{2}\right)$. The bottom panel shows the corresponding cross-sections with $x_{\text {FruR }}=10$ and $x_{\text {Gap } A}=10$ planes (arbitrary units).

As expected from previous considerations (see Sec. 2.2 of the main text), the surface is sigmoidal in shape, for both variables GapA and FruR and it can be approximated by a product of Hill functions, each one depending on a slow variable. In particular, the Hill function will be positive for FruR and negative for GapA, as evident from the slope of the curves in Fig. 17 (see panel $B$ ), with threshold concentrations $\theta_{\text {FruR }}^{1}, \theta_{\text {FruR }}^{2}$ and $\theta_{\text {Gap } A}^{1}, \theta_{\text {Gap A }}^{2}$, respectively.

As can be seen, the thresholds for free FruR, GapA and FruR are not independent since, by construction, they are supposed to lie on the same surface. Indeed, a change in the threshold concentration of free FruR causes a change in the corresponding threshold concentrations of GapA and FruR. Moreover, if $\theta_{\text {FruR free }}^{1}<\theta_{\text {FruR free }}^{2}$, the relative ordering between thresholds for FruR and GapA is imposed by the sigmoidal surface. In particular, the order is preserved for FruR i.e. $\theta_{F r u R}^{1}<\theta_{\text {FruR }}^{2}$ whereas it is reversed in the case of GapA, i.e. $\theta_{\text {Gap } A}^{1}>\theta_{\text {Gap } A}^{2}$, as shown in Fig. 17B. These dependencies between thresholds 
of variables controlling a coupling species thus reduce the number of possible orderings that have to be considered as model variants.

Further constraints come from biological knowledge on the system. For instance, the relative ordering of the focal points of GapA is known from the literature: gapA is transcribed from several promoters, among which promoter P1 (under the control of free FruR) is the most efficient (Thouvenot et al., 2004). We can therefore assume $\kappa_{\text {Gap } A}^{2} / \gamma_{\text {Gap } A}<\kappa_{\text {Gap } A}^{1} / \gamma_{\text {Gap } A}<\left(\kappa_{\text {Gap A }}^{1}+\right.$ $\left.\kappa_{\text {Gap A }}^{2}\right) / \gamma_{\text {Gap A }}$.

The variants of the models models $\mathcal{M}_{\text {glyco }}$ and $\mathcal{M}_{\text {neo }}$ studied in Sec. 3.5 of the main text have been generated along the lines sketched above. Despite the above mathematical and biological constraints, the number of possible variants remains too big for manual analysis (480 for our small example). We therefore opted for a random, uniform sampling of the ensemble of variants.

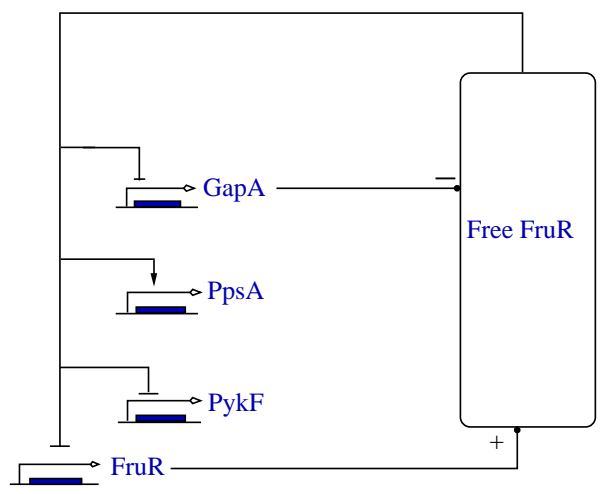

$$
\begin{aligned}
& \dot{x}_{\text {FruR }}=\kappa_{\text {FruR }}\left(1-s^{+}\left(x_{F r u R}, \theta_{\text {FruR }}^{3}\right) s^{-}\left(x_{\text {Gap A }}, \theta_{\text {Gap A }}^{3}\right)\right)-\gamma_{\text {FruR }} x_{\text {FruR }} \\
& \dot{x}_{\text {Gap } A}=\kappa_{\text {Gap } A}^{2}+\kappa_{\text {Gap A }}^{1}\left(1-s^{+}\left(x_{\text {FruR }}, \theta_{\text {FruR }}^{1}\right) s^{-}\left(x_{\text {Gap } A}, \theta_{\text {Gap } A}^{1}\right)\right)-\gamma_{\text {Gap A }} x_{\text {Gap } A} \\
& \dot{x}_{\text {Pps } A}=\kappa_{P p s A} s^{+}\left(x_{F r u R}, \theta_{F r u R}^{2}\right) s^{-}\left(x_{G a p A}, \theta_{\text {Gap } A}^{2}\right)-\gamma_{P p s A} x_{P p s A} \\
& \dot{x}_{P y k F}=\kappa_{P y k F}\left(1-s^{+}\left(x_{F r u R}, \theta_{F r u R}^{2}\right) s^{-}\left(x_{G a p A}, \theta_{\text {Gap } A}^{2}\right)\right)-\gamma_{P y k F} x_{P y k F}
\end{aligned}
$$

Figure 16: Network of indirect interactions mediated by the coupling species free FruR during gluconeogenesis and the corresponding PL model. 

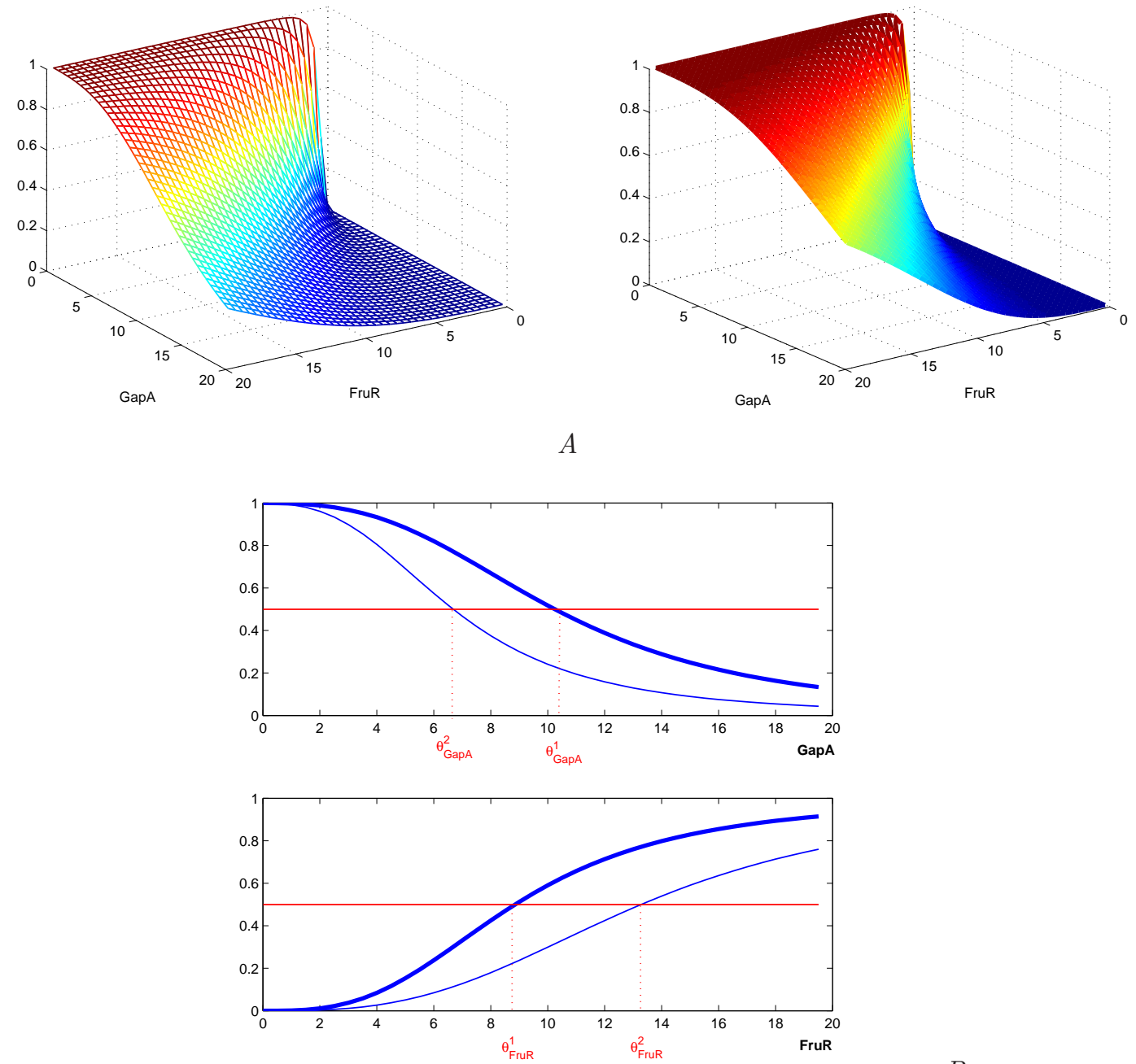

Figure 17: Plot of $h^{+}\left(x_{F r u R \text { free }}, \theta_{F r u R}\right.$ free $)$ as a function of $x_{F r u R}$ and $x_{G a p A}$ (total FruR and GapA concentrations in arbitrary units) for two different values of the threshold concentration $\theta_{\text {FruR free }}$ (left: $\theta_{\text {FruR free }}^{1}$, right: $\theta_{\text {FruR free }}^{2}$ ). B: Sections by the $x_{\text {FruR }}=10$ and $x_{\text {GapA }}=10$ planes, for the two values of $\theta_{\text {FruR free }}$. A red line at 0.5 defines the position of the corresponding thresholds concentrations for FruR and GapA $\left(\theta_{F r u R}^{1}, \theta_{F r u R}^{2}\right.$ and $\left.\theta_{\text {GapA }}^{1}, \theta_{\text {GapA }}^{2}\right)$. 


\section{References}

Baldazzi, V., Ropers, D., Markowicz, Y., Kahn, D., Geiselmann, J., and de Jong, H. 2010. The carbon assimilation network in Escherichia coli is densely connected and largely sign-determined by directions of metabolic fluxes. PloS Comput. Biol., 6(6):e1000812.

Batt, G., de Jong, H., Page, M., and Geiselmann, J. 2008. Symbolic reachability analysis of genetic regulatory networks using discrete abstractions. Automatica, 44(4):982-989.

Batt, G., Page, M., Cantone, I., Goessler, G., Monteiro, P., and de Jong, H. 2010. Efficient parameter search for qualitative models of regulatory networks using symbolic model checking. Bioinformatics, 26(18):i603-i610.

Bettenbrock, K., Fischer, S., Kremling, A., Jahreis, K., Sauter, T., and Gilles, E. 2005. A quantitative approach to catabolite repression in Escherichia coli. J. Biol. Chem., 281(5):2578-2584.

Bolouri, H. 2008. Computational Modeling of Gene Regulatory Networks - A Primer. Imperial College Press, London.

Brazhnik, P., de la Fuente, A., and Mendes, P. 2002. Gene networks: How to put the function in genomics. Trends Biotechnol., 20(11):467-472.

Chassagnole, C., Noisommit-Rizzi, N., Schmid, J., Mauch, K., and Reuss, M. 2002. Dynamic modeling of the central carbon metabolism of Escherichia coli. Biotechnol. Bioeng., 79(1):53-73.

Chaves, M., Sontag, E., and Albert, R. 2006. Methods for the robustness analysis for Boolean models. IET Syst. Biol., 153(4):154-167.

Covert, M., Knight, E., Reed, J., Herrgard, M., and Palsson, B. 2004. Integrating high-throughput and computational data elucidates bacterial networks. Nature, 429(6987):92-100.

Covert, M., Xiao, N., Chen, T., and Karr, J. 2008. Integrating metabolic, transcriptional regulatory and signal transduction models in Escherichia coli. Bioinformatics, 24:2044-2050.

Davidich, M. and Bornholdt, S. 2008. The transition from differential equations to Boolean networks: A case study in simplifying a regulatory network model. J. Theor. Biol., 255(3):269-277.

de Jong, H. 2002. Modeling and simulation of genetic regulatory systems: A literature review. J. Comput. Biol., 9(1):67-103.

de Jong, H., Geiselmann, J., Batt, G., Hernandez, C., and Page, M. 2004a. Qualitative simulation of the initiation of sporulation in B. subtilis. Bull. Math. Biol., 66(2):261-299.

de Jong, H., Geiselmann, J., Hernandez, C., and Page, M. 2003. Genetic Network Analyzer: Qualitative simulation of genetic regulatory networks. Bioinformatics, 19(3):336-344. 
de Jong, H., Gouzé, J.-L., Hernandez, C., Page, M., Sari, T., and Geiselmann, J. 2004b. Qualitative simulation of genetic regulatory networks using piecewiselinear models. Bull. Math. Biol., 66(2):301-340.

de Jong, H. and Page, M. 2008. Search for steady states of piecewise-linear differential equation models of genetic regulatory networks. ACM/IEEE Trans. Comput. Biol. Bioinfor., 5(2):208-222.

de Jong, H. and Ropers, D. 2006. Strategies for dealing with incomplete information in the modeling of molecular interaction networks. Brief. Bioinform., $7(4): 354-363$.

Fauré, A. and Thieffry, D. 2009. Logical modelling of cell cycle control in Eukaryotes: A comparative study. Mol. Biosyst., 5(12):1569-1581.

Glass, L. and Kauffman, S. 1973. The logical analysis of continuous non-linear biochemical control networks. J. Theor. Biol., 39(1):103-129.

Gutierrez-Ríos, R., Freyre-Gonzalez, J., Resendis, O., Collado-Vides, J., Saier, M., and Gosset, G. 2007. Identification of regulatory network topological units coordinating the genome-wide transcriptional response to glucose in Escherichia coli. BMC Microbiol., 7:53.

Hardiman, T., Lemuth, K., Reuss, M. K. M., and Siemann-Herzberg, M. 2007. Topology of the global regulatory network of carbon limitation in Escherichia coli. J. Biotechnol., 132(4):359-374.

Heinrich, R. and Schuster, S. 1996. The Regulation of Cellular Systems. Chapman \& Hall, New York.

Ishizuka, H., Hanamura, A., Kunimura, T., and Aiba, H. 1993. A lowered concentration of cAMP receptor protein caused by glucose is an important determinant for catabolite repression in Escherichia coli. Mol. Microbiol., $10(2): 341350$

Kao, K. C., Tran, L. M., and Liao, J. C. 2005. A global regulatory role of gluconeogenic genes in Escherichia coli revealed by transcriptome network analysis. J. Biol. Chem., 280(43):36079-36087.

Kappler, K., Edwards, R., and Glass, L. 2003. Dynamics in high-dimensional model gene networks. Signal Process., 83(4):789-798.

Karlebach, G. and Shamir, R. 2008. Modelling and analysis of gene regulatory networks. Nat. Rev. Mol. Cell Biol., 9(10):770-780.

Keseler, I. M., Bonavides-Martinez, C., Collado-Vides, J., Gama-Castro, S., Gunsalus, R. P., Johnson, D. A., Krummenacker, M., Nolan, L. M., Paley, S., Paulsen, I. T., Peralta-Gil, M., Santos-Zavaleta, A., Shearer, A. G., and Karp, P. D. 2009. EcoCyc: A comprehensive view of Escherichia coli biology. Nucleic Acids Res., 37:D464-470.

Khalil, H. 2001. Nonlinear Systems. Prentice Hall, Upper Saddle River, NJ, 3rd ed. edition. 
Klipp, E., Nordlander, B., Krüger, R., Gennemark, P., and Hohmann, S. 2005. Integrative model of the response of yeast to osmotic shock. Nat. Biotechnol., 23(8):975-982.

Kotte, O., Zaugg, J., and Heinemann, M. 2010. Bacterial adaptation through distributed sensing of metabolic fluxes. Mol. Syst. Biol., 6:355.

Kremling, A. 2007. Comment on mathematical models which describe transcription and calculate the relationship between mRNA and protein expression ratio. Biotechnol. Bioengin., 96(4):815-819.

Liu, M., Durfee, T., Cabrera, J., Zhao, K., Jin, D., and Blattner, F. 2005. Global transcriptional programs reveal a carbon source foraging strategy by Escherichia coli. J. Biol. Chem., 280(16):15921-15927.

Mandel, M. and Silhavy, T. 2005. Starvation for different nutrients in Escherichia coli results in differential modulation of RpoS levels and stability. J. Bacteriol., 187(2):434-442.

Monteiro, P., Ropers, D., Mateescu, R., Freitas, A., and de Jong, H. 2008. Temporal logic patterns for querying dynamic models of cellular interaction networks. Bioinformatics, 24(16):i227-i233.

Morris, M., Saez-Rodriguez, J., Sorger, P., and Lauffenburger, D. 2010. Logicbased models for the analysis of cell signaling networks. Biochemistry, 49(15):3216-3225.

Nanchen, A., Schicker, A., Revelles, O., and Sauer, U. 2008. Cyclic AMPdependent catabolite repression is the dominant control mechanism of metabolic fluxes under glucose limitation in Escherichia coli. J. Bacteriol., 190(7):2323-2330

Oh, M.-K., Rohlin, L., Kao, K. C., and Liao, J. C. 2002. Global expression profiling of acetate-grown Escherichia coli. J. Biol. Chem., 277(15):1317513183.

Peng, L. and Shimizu, K. 2003. Global metabolic regulation analysis for Escherichia coli K12 based on protein expression by 2-dimensional electrophoresis and enzyme activity measurement. Appl. Microbiol. Biotechnol., 61(2):163-178.

Plahte, E., Mestl, T., and Omholt, S. 1998. A methodological basis for description and analysis of systems with complex switch-like interactions. J. Math. Biol., 36(4):321-348.

Polynikis, A., Hogan, S., and di Bernardo, M. 2009. Comparing different ODE modelling approaches for gene regulatory networks. J. Theor. Biol., 261(4):511-30.

Reder, C. 1988. Metabolic control theory: a structural approach. J. Theor. Biol., 135(2):175-201. 
Ropers, D., Baldazzi, V., and de Jong, H. 2011. Model reduction using piecewiselinear approximations preserves dynamic properties of the carbon starvation response in Escherichia coli. ACM/IEEE Trans. Comput. Biol. Bioinform., 8(1):166-181.

Ropers, D., de Jong, H., Page, M., Schneider, D., and Geiselmann, J. 2006. Qualitative simulation of the carbon starvation response in Escherichia coli. Biosystems, 84(2):124-152.

Saier, M. J., Ramseier, T., and J., R. 1996. Regulation of carbon utilization. In: Neidhardt, F., Curtiss III, R., Ingraham, J., Lin, E., Low, K., Magasanik, B., Reznikoff, W., Riley, M., Schaechter, M., and Umbarger, H. (Editors), Escherichia coli and Salmonella: Cellular and Molecular Biology, pages 132543. ASM Press, Washington D.C.

Sarkar, D., Siddiquee, K., Araúzo-Bravo, M., Oba, T., and Shimizu, K. 2008. Effect of cra gene knockout together with edd and $i c l R$ genes knockout on the metabolism in Escherichia coli. Arch. Microbiol., 190:558-571.

Sepulchre, J.-A., Reverchon, S., and Nasser, W. 2007. Modeling the onset of virulence in a pectinolytic bacterium. J. Theor. Biol., 44(2):239-257.

Shlomi, T., Eisenberg, Y., Sharan, R., and Ruppin, E. 2007. A genome-scale computational study of the interplay between transcriptional regulation and metabolism. Mol. Syst. Biol., 3:101.

Snoussi, E. 1989. Qualitative dynamics of piecewise-linear differential equations: A discrete mapping approach. Dynam. Stab. Syst., 4(3-4):189-207.

Thouvenot, B., Charpentier, B., and Branlant, C. 2004. The strong efficiency of the Escherichia coli gapA P1 promoter depends on a complex combination of functional determinants. Biochem. J., 383:371-382.

Usseglio Viretta, A. and Fussenegger, M. 2004. Modeling the quorum sensing regulatory network of human-pathogenic Pseudomonas aeruginosa. Biotechnol. Prog., 20(3):670-678.

Venturi, V. 2003. Control of rpoS transcription in Escherichia coli and Pseudomonas: why so different? Mol. Microbiol., 49:1-9.

Wittmann, D., Krumsiek, J., Saez-Rodriguez, J., Lauffenburger, D., Klamt, S., and Theis, F. 2009. Transforming Boolean models to continuous models: Methodology and application to T-cell receptor signaling. BMC Syst. Biol., $3: 98$.

Zgurskaya, H., Keyhan, M., and Matin, A. 1997. The $\sigma^{s}$ level in starving Escherichia coli cells increases solely as a result of its increased stability, despite decreased synthesis. Mol. Microbiol., 24(3):643-652.

\section{Contents}


2 Methods 6

2.1 Metabolic coupling in gene regulatory networks . . . . . . . . 6

2.2 Including metabolic coupling in dvnamic modelsof gene regulatory networks 9

2.3 Qualitative modeling of gene regulatory networks . . . . . . . 10

3 Results and discussion 13

3.1 Metabolic coupling in network controlling carbon assimilationin E. coli 13

3.2 Qualitative modeling of carbon assimilation network . . . . . . 15

3.3 Steadv states of network during growth on glucose and acetate . 17

3.4 Dvnamic response of gene expression to diauxic shift . . . . . . 20

3.5 Robustness analysis to parameter changes . . . . . . . . . . . 22

\begin{tabular}{|lll}
4 & Conclusions & 23
\end{tabular}

A Kinetic model of subnetwork controlling Crp activation 25

\begin{tabular}{ll}
\hline B PL models $\mathcal{M}_{\text {glyen }}$ and $\mathcal{M}_{\text {ned }}$ & 27
\end{tabular}

\begin{tabular}{lll}
\hline C PL models of $\mathcal{M}^{0}$ reference networks & 27
\end{tabular}

D Model variants of $\mathcal{M}_{\text {glyco }}$ and $\mathcal{M}_{\text {neo }}$ used for robustness tests 36 
Centre de recherche INRIA Grenoble - Rhône-Alpes 655, avenue de l'Europe - 38334 Montbonnot Saint-Ismier (France)

Centre de recherche INRIA Bordeaux - Sud Ouest : Domaine Universitaire - 351, cours de la Libération - 33405 Talence Cedex Centre de recherche INRIA Lille - Nord Europe : Parc Scientifique de la Haute Borne - 40, avenue Halley - 59650 Villeneuve d'Ascq Centre de recherche INRIA Nancy - Grand Est : LORIA, Technopôle de Nancy-Brabois - Campus scientifique 615, rue du Jardin Botanique - BP 101 - 54602 Villers-lès-Nancy Cedex

Centre de recherche INRIA Paris - Rocquencourt : Domaine de Voluceau - Rocquencourt - BP 105 - 78153 Le Chesnay Cedex

Centre de recherche INRIA Rennes - Bretagne Atlantique : IRISA, Campus universitaire de Beaulieu - 35042 Rennes Cedex Centre de recherche INRIA Saclay - Île-de-France : Parc Orsay Université - ZAC des Vignes : 4, rue Jacques Monod - 91893 Orsay Cedex Centre de recherche INRIA Sophia Antipolis - Méditerranée : 2004, route des Lucioles - BP 93 - 06902 Sophia Antipolis Cedex 